\title{
Harnack's inequality for doubly nonlinear equations of slow diffusion type
}

\author{
Verena Bögelein ${ }^{1}$ (D) Andreas Heran ${ }^{2} \cdot$ Leah Schätzler $^{1}$ (D) Thomas Singer $^{3}$
}

Received: 14 June 2020 / Accepted: 24 June 2021

(c) The Author(s) 2021

\section{Abstract}

In this article we prove a Harnack inequality for non-negative weak solutions to doubly nonlinear parabolic equations of the form

$$
\partial_{t} u-\operatorname{div} \mathbf{A}\left(x, t, u, D u^{m}\right)=\operatorname{div} F,
$$

where the vector field $\mathbf{A}$ fulfills $p$-ellipticity and growth conditions. We treat the slow diffusion case in its full range, i.e. all exponents $m>0$ and $p>1$ with $m(p-1)>1$ are included in our considerations.

Mathematics Subject Classification 35K55 $\cdot 35 \mathrm{~K} 65 \cdot 35 \mathrm{~B} 45 \cdot 35 \mathrm{~B} 65$

\section{Introduction and results}

Let $\Omega \subset \mathbb{R}^{n}, n \geq 2$, be a bounded open domain and $(0, T)$ with $0<T<\infty$ a finite time interval. In the following, $\Omega_{T}:=\Omega \times(0, T)$ denotes the related space-time cylinder. The prototype of the doubly nonlinear equations we are concerned with is

Communicated by N. Trudinger.

$\triangle$ Verena Bögelein

verena.boegelein@sbg.ac.at

Andreas Heran

heran@math.fau.de

Leah Schätzler

leahanna.schaetzler@sbg.ac.at

Thomas Singer

thomas.singer@teambank.de

1 Fachbereich Mathematik, Universität Salzburg, Hellbrunner Str. 34, 5020 Salzburg, Austria

2 Department of Data Science, Friedrich-Alexander-Universität Erlangen-Nürnberg, Cauerstrasse 11, 91058 Erlangen, Germany

3 TeamBank AG, Beuthener Strasse 25, 90471 Nürnberg, Germany 


$$
\partial_{t} u-\operatorname{div}\left(\left|D u^{m}\right|^{p-2} D u^{m}\right)=0 \quad \text { in } \Omega_{T}
$$

for non-negative solutions $u: \Omega_{T} \rightarrow \mathbb{R}_{\geq 0}$ with parameters $m \in(0, \infty)$ and $p \in(1, \infty)$. If $m=1,(1.1)$ reduces to the parabolic $p$-Laplace equation, whereas for $p=2$ we retrieve the porous medium equation. Doubly nonlinear equations of type (1.1) are classified as doubly degenerate if $m>1$ and $p>2$, singular-degenerate if $m>1$ and $p \in(1,2)$, degeneratesingular if $m \in(0,1)$ and $p>2$ and doubly singular if $m \in(0,1)$ and $p \in(1,2)$. Furthermore, depending on the behavior of solutions, we distinguish between slow diffusion equations with $m(p-1)>1$ and fast diffusion equations with $m(p-1)<1$. The qualitative difference between both cases stems from the fact that in the former one solutions might have a compact support, while this is not possible in the latter one. In the present paper, we treat the complete slow diffusion range $p(m-1)>1$, which includes the doubly degenerate case and the singular-degenerate and degenerate-singular slow diffusion case.

In the literature, (1.1) often appears in equivalent forms; cf. [17-20,28,33]. More precisely, we note that formally (1.1) is a transformation of

$$
\partial_{t} u^{\widehat{m}}-\operatorname{div}\left(|D u|^{p-2} D u\right)=0
$$

with $\widehat{m}:=\frac{1}{m}$ and

$$
\partial_{t} u-c(\ell, p) \operatorname{div}\left(u^{\ell}|D u|^{p-2} D u\right)=0
$$

where $\ell:=(m-1)(p-1)$. These representations of (1.1) can be shown to be equivalent. Let us also note that for $m>1$ there are two different notions of weak solutions to the porous medium equation and doubly nonlinear equations in the literature. The first one assumes that $u^{\frac{m+1}{2}}$ is weakly differentiable with respect to the space variable, whereas the second one claims this for $u^{m}$ (in the case $m<1$ only the latter one makes sense). For the prototype porous medium equation the equivalence of both notions of solutions has been shown in [6]. It is still an open problem if the same is true for doubly nonlinear equations and porous medium type equations with a general structure.

Harnack estimates play a crucial role in the regularity theory of partial differential equations. In the elliptic setting, essential contributions are due to Moser [25] for linear elliptic equations and Serrin [29] and Trudinger [31] for quasilinear elliptic equations. In the parabolic setting, the first results have been obtained by Hadamard [16] and Pini [27] for non-negative solutions of the heat equation. For the heat equation Harnack's inequality takes the form

$$
c^{-1} \sup _{B_{\varrho}\left(x_{o}\right)} u\left(\cdot, t_{o}-\varrho^{2}\right) \leq u\left(x_{o}, t_{o}\right) \leq c \sup _{B_{\varrho}\left(x_{o}\right)} u\left(\cdot, t_{o}+\varrho^{2}\right)
$$

with waiting time $\varrho^{2}$. Moser [26] showed that this result is true for linear parabolic equations as well and demonstrated the necessity of the waiting time. Later, Trudinger [32] proved Harnack inequalities for quasilinear parabolic equations and the homogeneous doubly nonlinear equation

$$
\partial_{t}\left(u^{p-1}\right)-\operatorname{div}\left(|D u|^{p-2} D u\right)=0
$$

with $p>1$. Using an approach based on mean value inequalities for suitable De Giorgi classes, Gianazza and Vespri [14] gave a proof that extends to more general operators $A(x, t, u, D u)$ instead of $|D u|^{p-2} D u$. Finally, simplifying an approach originally introduced by Moser, Kinnunen \& Kuusi [22] obtained Harnack's inequality for the homogeneous doubly nonlinear equation, where the Lebesgue measure is replaced by a more general Borel measure. In the case of non-homogeneous nonlinear equations, the situation is more involved. 
DiBenedetto [7] proved that non-negative weak solutions of the parabolic $p$-Laplace equation and the porous medium equation satisfy an intrinsic Harnack inequality of the form

$$
c^{-1} \sup _{B_{\varrho}\left(x_{o}\right)} u\left(\cdot, t_{o}-t_{w}\right) \leq u\left(x_{o}, t_{o}\right) \leq c \inf _{B_{\varrho}\left(x_{o}\right)} u\left(\cdot, t_{o}+t_{w}\right)
$$

with $t_{w}=c u\left(x_{o}, t_{o}\right)^{2-p} \varrho^{p}$ for the parabolic $p$-Laplace equation and $t_{w}=c u\left(x_{o}, t_{o}\right)^{1-m} \varrho^{2}$ for the porous medium equation. These Harnack inequalities are called intrinsic, because the waiting times depend on the solution itself. Loosely speaking, solutions of non-homogeneous equations behave like solutions of the heat equation in an intrinsic time scale. A counterexample [11] shows that a Harnack estimate with $t_{w}$ independent of $u$ is false. Since the proof in [7] relies on comparison with explicit solutions, it cannot be adapted for general quasilinear equations. Nearly 20 years later, this problem was overcome by DiBenedetto, Gianazza \& Vespri [9], whose proof only uses measure theoretical tools. The main novelty is the socalled Expansion of Positivity. The same method was used by Kuusi [23] to obtain weak Harnack estimates for super-solutions of nonlinear degenerate parabolic equations. For an extensive overview regarding the parabolic $p$-Laplace equation and the porous medium equation with the definition of weak solution involving $u^{\frac{m+1}{2}}$, we refer to the monograph [10] by DiBenedetto, Gianazza and Vespri and the survey [11] by Düzgün, Fornaro and Vespri. Harnack's inequality for the prototype doubly nonlinear equation

$$
\partial_{t} u-\operatorname{div}\left(|u|^{m-1}|D u|^{p-2} D u\right)=0
$$

has first been proved by Vespri [33] for the full range of parameters $p>1$ and $m+p>$ $\max \left\{2,3-\frac{p}{n}\right\}$. The proof uses explicit constructions involving the Barenblatt solution and therefore cannot be applied to more general structures. For the doubly degenerate case Fornaro and Sosio [12] generalized the result to weak solutions of

$$
\partial_{t} u-\operatorname{div} \mathbf{A}(x, t, u, D u)=\mathbf{B}(x, t, u, D u),
$$

where the operators $\mathbf{A}$ and $\mathbf{B}$ fulfill the conditions

$$
\left\{\begin{aligned}
\mathbf{A}(x, t, u, \xi) \cdot \xi & \geq c_{0} \Phi(|u|)|\xi|^{p}-c^{p} \\
|\mathbf{A}(x, t, u, \xi)| & \leq c_{1} \Phi(|u|)|\xi|^{p-1}+c^{p-1} \Phi(|u|)^{\frac{1}{p}} \\
|\mathbf{B}(x, t, u, \xi)| & \leq c_{2} \Phi(|u|)|\xi|^{p-1}+c_{2} c^{p-1} \Phi(|u|)^{\frac{1}{p}}
\end{aligned}\right.
$$

with $p \geq 2$, positive constants $c_{0}, c_{1}, c_{2}, c$ and a function $\Phi$ satisfying an $(m-1)$-growth condition with $m \geq 1$. They used a definition of weak solution involving $u^{\frac{m+1}{2}}$. A weak Harnack inequality for super-solutions can be found in [24]. For the case of fast diffusion equations, we refer to the articles by Fornaro, Sosio and Vespri [13] and Vespri and Vestberg [34].

In this paper we prove Harnack's inequality for the entire slow diffusion range and thereby close the gap for the by now missing singular-degenerate and degenerate-singular slow diffusion cases. Furthermore, we work with a definition of weak solution involving $u^{m}$, which is new even for the doubly degenerate case and the slow diffusion porous medium equation.

\subsection{Setting}

We consider non-negative weak solutions to the doubly nonlinear equation

$$
\partial_{t} u-\operatorname{div} \mathbf{A}\left(x, t, u, D u^{m}\right)=\operatorname{div} F \quad \text { in } \Omega_{T}
$$


with $m>0$. For the vector field A: $\Omega_{T} \times \mathbb{R} \times \mathbb{R}^{n} \rightarrow \mathbb{R}^{n}$ we assume that $\mathbf{A}$ is measurable with respect to $(x, t) \in \Omega_{T}$ for all $(u, \xi) \in \mathbb{R} \times \mathbb{R}^{n}$ and continuous with respect to $(u, \xi)$ for a.e. $(x, t) \in \Omega_{T}$. Moreover, we assume that $\mathbf{A}$ satisfies the following growth and ellipticity conditions

$$
\mathbf{A}(x, t, u, \xi) \cdot \xi \geq v|\xi|^{p} \quad \text { and } \quad|\mathbf{A}(x, t, u, \xi)| \leq L|\xi|^{p-1},
$$

for $p>1$ and structure constants $0<v \leq L<\infty$. We demand that

$$
F \in L^{\sigma}\left(\Omega_{T}, \mathbb{R}^{n}\right)
$$

for some $\sigma>\frac{n+p}{p-1}$ and that the parameters $m$ and $p$ satisfy $m(p-1)>1$ which means that we are in the slow diffusion range. In the following we abbreviate

$$
d:=m(p-1)-1>0 .
$$

We now give the precise definition of weak solution to (1.3) that we use throughout the paper.

Definition 1.1 Assume that the vector field A satisfies (1.4). A non-negative measurable function $u: \Omega_{T} \rightarrow \mathbb{R}_{\geq 0}$ in the class

$$
u \in C^{0}\left((0, T) ; L_{\mathrm{loc}}^{m+1}(\Omega)\right) \text { with } u^{m} \in L_{\mathrm{loc}}^{p}\left(0, T ; W_{\mathrm{loc}}^{1, p}(\Omega)\right)
$$

is a non-negative weak sub(super)-solution to the doubly nonlinear equation (1.3) if and only if the identity

$$
\iint_{\Omega_{T}}\left[-u \cdot \partial_{t} \varphi+\mathbf{A}\left(x, t, u, D u^{m}\right) \cdot D \varphi\right] \mathrm{d} x \mathrm{~d} t \stackrel{(\geq)}{\leq} \iint_{\Omega_{T}} F \cdot D \varphi \mathrm{d} x \mathrm{~d} t
$$

holds true for any testing function $\varphi \in C_{0}^{\infty}\left(\Omega_{T}, \mathbb{R}_{\geq 0}\right)$. If $u$ is a weak sub- and super-solution it is called a weak solution.

We are now in the position to formulate the main result of our paper:

Theorem 1.2 Let $m>0, p>1$ with $m(p-1)>1$ and $u$ be a continuous, non-negative, weak solution to (1.3) in the sense of Definition 1.1, where the vector field $\mathbf{A}$ satisfies (1.4) and $F$ satisfies (1.5). Moreover, let $\left(x_{o}, t_{o}\right) \in \Omega_{T}$ such that $u\left(x_{o}, t_{o}\right)>0$. Then, there exist constants $c_{o}, \gamma>1$ depending only on $n, m, p, L, v$ and $\sigma$ such that for all cylinders $B_{9 \varrho}\left(x_{o}\right) \times\left(t_{o}-4 \theta \varrho^{p}, t_{o}+4 \theta \varrho^{p}\right) \Subset \Omega_{T}$, with

$$
\theta=\left(\frac{c_{o}}{u\left(x_{o}, t_{o}\right)}\right)^{d}
$$

we either have

$$
\|F\|_{L^{\sigma}\left(\Omega_{T}\right)} \varrho^{p-1-\frac{n+p}{\sigma}} \geq \frac{1}{\gamma} u\left(x_{o}, t_{o}\right)^{d+1-\frac{d}{\sigma}}
$$

or

$$
\left(2 \gamma^{2}\right)^{-1} \sup _{B_{\varrho}\left(x_{o}\right)} u\left(\cdot, t_{o}-(2 \gamma)^{-d} \theta \varrho^{p}\right) \leq u\left(x_{o}, t_{o}\right) \leq \gamma \inf _{B_{\varrho}\left(x_{o}\right)} u\left(\cdot, t_{o}+\theta \varrho^{p}\right) .
$$

Note that the continuity assumption in Theorem 1.2 is not restrictive. The Harnack inequality continues to hold for a.e. point $\left(x_{o}, t_{o}\right) \in \Omega_{T}$ if we state it for an arbitrary non-negative, weak solution to (1.3). However, for the sake of a neater exposition of the result, we prefer to state it for continuous solutions. 


\subsection{Plan of the paper}

In Sect. 2 we collect some auxiliary tools. Using $u^{m}-a^{m}$ for some level $a$ as test function in (a mollified version of) the definition of weak sub- and super-solutions, we derive certain Caccioppoli inequalities in Sect. 3. For convenience of the reader we state all intermediate results for weak sub- respectively super-solutions instead of weak solutions, so that it becomes clear what the minimal assumptions are. Next, in Sect. 4 we show that weak sub-solutions to (1.3) are locally bounded and give a quantitative estimate. In Sect. 5 we prove so-called De Giorgi type lemmas. Loosely speaking, the first lemma shows that if a super-solution $u$ to (1.3) is smaller than some level $M$ only on a small enough proportion of a suitable cylinder, then $u$ is larger than $\frac{M}{2}$ a.e. on a smaller cylinder contained in the first one. The second lemma gives an analogous statement for sub-solutions in the case that $u$ is larger than a fixed level only on a small enough proportion of the bigger cylinder and consequently smaller than a fraction of the level on the smaller cylinder. The proofs of the statements rely in particular on the Caccioppoli estimates. In Sect. 6 we prove Expansion of Positivity of non-negative weak super-solutions. The conclusion of the section is that if

$$
\left|\left\{u\left(t_{o}\right) \geq M\right\} \cap B_{\varrho}\left(x_{o}\right)\right| \geq \alpha\left|B_{\varrho}\left(x_{o}\right)\right|
$$

for a level $M>0, \alpha \in(0,1)$ and a suitable ball $B_{\varrho}\left(x_{o}\right)$, then $u \geq \kappa M$ a.e. in $B_{2 \varrho}\left(x_{o}\right) \times\left(t_{o}+\right.$ $\left.\frac{1}{2} b(\kappa M)^{-d} \varrho^{p}, t_{o}+b(\kappa M)^{-d} \varrho^{p}\right]$. Here, the constants $b, \kappa \in(0,1)$ depend only on the data and $\alpha$. In the proof, the Caccioppoli estimates and the first De Giorgi type lemma are used. Finally, in Sect. 7 we deduce the intrinsic Harnack inequality stated in Theorem 1.2. To show the forward inequality, i.e. the second inequality in (1.8), after a transformation we use the second De Giorgi type lemma and iteratively apply Expansion of positivity. Subsequently, we prove that the forward inequality implies the backward Harnack inequality, i.e. the first inequality in (1.8). Actually, a more general version of the backward Harnack inequality is shown in Sect. 7.

\section{Preliminaries}

\subsection{Notation}

First, we introduce some notation used throughout the paper. For functions defined on $\Omega_{T}$, we denote the time slice at time $t \in(0, T)$ by $v(t):=v(\cdot, t)$. For $z_{o}=\left(x_{o}, t_{o}\right) \in \mathbb{R}^{n} \times \mathbb{R}$ we define space-time cylinders

$$
\begin{aligned}
& Q_{\varrho, \theta}^{-}\left(z_{o}\right):=B_{\varrho}\left(x_{o}\right) \times \Lambda_{\theta}^{-}\left(t_{o}\right):=B_{\varrho}\left(x_{o}\right) \times\left(t_{o}-\theta, t_{o}\right] \\
& Q_{\varrho, \theta}^{+}\left(z_{o}\right):=B_{\varrho}\left(x_{o}\right) \times \Lambda_{\theta}^{+}\left(t_{o}\right):=B_{\varrho}\left(x_{o}\right) \times\left(t_{o}, t_{o}+\theta\right]
\end{aligned}
$$

with a radius $\varrho>0$ and time length $\theta>0$ and let

$$
Q_{\varrho, \theta}\left(z_{o}\right):=Q_{\varrho, \theta}^{-}\left(z_{o}\right) \cup Q_{\varrho, \theta}^{+}\left(z_{o}\right)
$$

As usual, we let

$$
(u-a)_{+}:=\max \{u-a, 0\}, \quad(u-a)_{-}:=\max \{-(u-a), 0\},
$$

for $u, a \in \mathbb{R}$. Furthermore, for $u, a \geq 0$ we define the boundary term

$$
\mathfrak{b}\left[u^{m}, a^{m}\right]:=\frac{m}{m+1}\left(a^{m+1}-u^{m+1}\right)-u \cdot\left(a^{m}-u^{m}\right) .
$$




\subsection{Mollification in time}

Since weak solutions do not possess a time derivative in general we have to use mollification. To this end, for $v \in L^{1}\left(\Omega_{T}, \mathbb{R}^{N}\right)$ and $h>0$ we define the following mollification in time

$$
\llbracket v \rrbracket_{h}:=\frac{1}{h} \int_{0}^{t} e^{\frac{s-t}{h}} v(x, s) \mathrm{d} s,
$$

which formally satisfies the ordinary differential equation

$$
\partial_{t} \llbracket v \rrbracket_{h}=-\frac{1}{h}\left(\llbracket v \rrbracket_{h}-v\right) .
$$

Basic properties of $\llbracket \cdot \rrbracket_{h}$ are provided in the following lemma. For its proof and further information, we refer to [21, Lemma 2.2] and [5, Appendix B].

Lemma 2.1 Suppose that $X$ is a separable Banach space. If $v \in L^{r}(0, T ; X)$ for some $r \geq 1$, then the mollification $\llbracket v \rrbracket_{h}$ defined in (2.2) fulfills $\llbracket v \rrbracket_{h} \in L^{r}(0, T ; X)$ and for any $t_{o} \in(0, T]$ there holds

$$
\left\|\llbracket v \rrbracket_{h}\right\|_{L^{r}\left(0, t_{o} ; X\right)} \leq\|v\|_{L^{r}\left(0, t_{o} ; X\right)} .
$$

Moreover, in the case $r<\infty$ we have $\llbracket \cdot \rrbracket_{h} \rightarrow v$ in $L^{r}(0, T ; X)$ as $h \downarrow 0$.

Using the same technique as in [30, Lemma 3.6], we conclude that any sub(super)-solution to (1.3) in the sense of Definition 1.1 satisfies the mollified version of (1.6),

$$
\begin{aligned}
& \iint_{\Omega_{T}}\left[\partial_{t} \llbracket u \rrbracket_{h} \varphi+\llbracket \mathbf{A}\left(x, t, u, D u^{m}\right) \rrbracket_{h} \cdot D \varphi\right] \mathrm{d} x \mathrm{~d} t \\
& \quad(\geq)) \iint_{\Omega_{T}} \llbracket F \rrbracket_{h} \cdot D \varphi \mathrm{d} x \mathrm{~d} t+\frac{1}{h} \int_{\Omega} u(0) \int_{0}^{T} e^{-\frac{s}{h}} \varphi \mathrm{d} s \mathrm{~d} x
\end{aligned}
$$

for any $\varphi \in C_{0}^{\infty}\left(\Omega_{T}, \mathbb{R}_{\geq 0}\right)$.

\subsection{Transformation}

The following Lemma is an easy consequence of a change of variables.

Lemma 2.2 Let $T>0, I \subset \mathbb{R}$ be an open interval and $\Phi: I \rightarrow(0, T)$ an increasing $C^{\infty}$-diffeomorphism. Then, $u$ is a weak sub(super)-solution to (1.3) associated to $\mathbf{A}, F$ in $B_{Q} \times(0, T)$ if and only if the function $w(x, \tau):=u(x, \Phi(\tau))$ is a sub(super)-solution to (1.3) associated to the vector field

$$
\widetilde{\mathbf{A}}(x, \tau, u, \xi):=\Phi^{\prime}(\tau) \mathbf{A}(x, \Phi(\tau), u, \xi)
$$

and right-hand side $\widetilde{F}(x, \tau):=\Phi^{\prime}(\tau) F(x, \Phi(\tau))$ in $B_{\varrho} \times I$.

The next Lemma shows that the product of a non-negative weak super-solution $u$ with a non-decreasing $C^{1}$-function $\gamma$ is a super-solution to a modified equation. A similar argument has already been used in [9].

Lemma 2.3 Let $\Omega \subset \mathbb{R}^{n}$ be bounded and open and $I \subset \mathbb{R}$ an open interval. Assume that $u$ is a non-negative weak super-solution to (1.3) in $\Omega \times I$ associated to $\mathbf{A}, F$ and $\gamma \in C^{1}(I) \cap C^{0}(\bar{I})$ is non-decreasing and satisfies $\frac{1}{C} \leq \gamma \leq C$ on I for a constant $C \geq 1$. Then, the function $\tilde{u}:=\gamma u$ is a non-negative weak super-solution to (1.3) in $\Omega \times I$ associated to the vector-field

$$
\widetilde{\mathbf{A}}(x, t, u, \xi):=\gamma(\tau) \mathbf{A}\left(x, t, \frac{u}{\gamma(t)}, \frac{\xi}{\gamma(t)^{m}}\right)
$$


and inhomogeneity $\widetilde{F}:=\gamma F$.

Proof In the following we abbreviate $\Omega_{I}:=\Omega \times I$. Let $\varphi \in C_{0}^{\infty}\left(\Omega_{I}, \mathbb{R}_{\geq 0}\right)$. Then $\gamma \varphi \in C_{0}^{1}\left(\Omega_{I}, \mathbb{R}_{\geq 0}\right)$. By assumption $\gamma^{\prime}, u$ and therefore also $\llbracket u \rrbracket_{h}$ are non-negative. By an approximation argument we may use $\gamma \varphi$ as testing function in the mollified weak formulation (2.4) on the interval $I$ instead of $(0, T)$. This leads to

$$
\begin{aligned}
-\iint_{\Omega_{I}} \llbracket u \rrbracket_{h} \gamma \partial_{t} \varphi \mathrm{d} x \mathrm{~d} t= & \iint_{\Omega_{I}} \partial_{t}\left(\llbracket u \rrbracket_{h} \gamma\right) \varphi \mathrm{d} x \mathrm{~d} t=\iint_{\Omega_{I}}\left[\partial_{t} \llbracket u \rrbracket_{h} \gamma \varphi+\llbracket u \rrbracket_{h} \gamma^{\prime} \varphi\right] \mathrm{d} x \mathrm{~d} t \\
\geq & \iint_{\Omega_{I}} \partial_{t} \llbracket u \rrbracket_{h} \gamma \varphi \mathrm{d} x \mathrm{~d} t \\
\geq & -\iint_{\Omega_{I}} \llbracket \mathbf{A}\left(x, t, u, D u^{m}\right) \rrbracket_{h} \cdot D(\gamma \varphi) \mathrm{d} x \mathrm{~d} t \\
& +\iint_{\Omega_{I}} \llbracket F \rrbracket_{h} \cdot D(\gamma \varphi) \mathrm{d} x \mathrm{~d} t+\frac{1}{h} \int_{\Omega} u(0) \int_{I} e^{-\frac{s}{h}}(\gamma \varphi) \mathrm{d} s \mathrm{~d} x .
\end{aligned}
$$

Passing to the limit $h \downarrow 0$ with the help of Lemma 2.1 and taking into account that $\operatorname{spt}(\gamma \varphi)$ is compact in the last term on the right-hand side, this leads to

$$
\iint_{\Omega_{I}}\left[-\gamma u \partial_{t} \varphi+\gamma \mathbf{A}\left(x, t, u, D u^{m}\right) \cdot D \varphi\right] \mathrm{d} x \mathrm{~d} t \geq \iint_{\Omega_{I}} \gamma F \cdot D \varphi \mathrm{d} x \mathrm{~d} t
$$

for every $\varphi \in C_{0}^{\infty}\left(\Omega_{I}, \mathbb{R}_{\geq 0}\right)$, which is in view of the definition of $\tilde{u}$ and $\widetilde{F}$ equivalent to

$$
\iint_{\Omega_{I}}\left[-\tilde{u} \partial_{t} \varphi+\gamma \mathbf{A}\left(x, t, \frac{\tilde{u}}{\gamma}, \frac{D \tilde{u}^{m}}{\gamma^{m}}\right) \cdot D \varphi\right] \mathrm{d} x \mathrm{~d} t \geq \iint_{\Omega_{I}} \widetilde{F} \cdot D \varphi \mathrm{d} x \mathrm{~d} t .
$$

Recalling the definition of $\widetilde{\mathbf{A}}$, this yields the claim.

Combining the last two lemmata leads to the following statement, which is used in the proof of the expansion of positivity.

Corollary 2.4 Let $T>0$ and $u$ a non-negative weak super-solution to (1.3) in $B_{Q} \times(0, T)$ associated to $\mathbf{A}$ and $F$. Further, assume that $I \subset \mathbb{R}$ is an open interval, that $\Phi: I \rightarrow(0, T)$ is an increasing $C^{\infty}$-diffeomorphism and that $\gamma \in C^{1}(I) \cap C^{0}(\bar{I})$ is non-decreasing and satisfies $\frac{1}{C} \leq \gamma \leq C$ on I for some constant $C \geq 1$. Then, the function $v(x, \tau):=\gamma(\tau)$. $u(x, \Phi(\tau))$ is a non-negative weak super-solution to (1.3) in $B_{\varrho} \times I$ associated to the vectorfield

$$
\widehat{\mathbf{A}}(x, \tau, u, \xi):=\gamma(\tau) \Phi^{\prime}(\tau) \mathbf{A}\left(x, \Phi(\tau), \frac{u}{\gamma(\tau)}, \frac{\xi}{\gamma(\tau)^{m}}\right)
$$

and inhomogeneity $\widehat{F}(x, \tau):=\gamma(\tau) \Phi^{\prime}(\tau) F(x, \Phi(\tau))$.

\subsection{Auxiliary lemmata}

For a function $v \in W^{1,1}$ and $k<\ell$ the next lemma gives a local estimate for the product of the measures of superlevel sets $\{v>\ell\}$ and sublevel sets $\{v<k\}$ in terms of the $L^{1}$-norm of $D v$ on the intersection of their complements, cf. [8, Chap. I.2, Lemma 2.2 and Remark 2.3].

Lemma 2.5 Let $v \in W^{1,1}\left(B_{\varrho}\left(x_{o}\right)\right)$ and $k, \ell \in \mathbb{R}$ with $k<\ell$. Then, there exists a constant $c$ depending on $n$ such that

$$
(\ell-k)\left|B_{\varrho}\left(x_{o}\right) \cap\{v<k\}\right| \leq \frac{c \varrho^{n+1}}{\left|B_{\varrho}\left(x_{o}\right) \cap\{v>\ell\}\right|} \int_{B_{\varrho}\left(x_{o}\right) \cap\{k<v<\ell\}}|D v| \mathrm{d} x .
$$


The following lemma can be found in the literature; cf. [1, Lemma 2.2] for $\alpha \in(0,1)$ and [15, inequality (2.4)] for $\alpha>1$.

Lemma 2.6 For any $\alpha>0$, there exists a constant $c=c(\alpha)$ such that, for all $a, b \geq 0$, the following inequality holds true:

$$
\frac{1}{c}\left|b^{\alpha}-a^{\alpha}\right| \leq(|a|+|b|)^{\alpha-1}|b-a| \leq c\left|b^{\alpha}-a^{\alpha}\right| .
$$

The next lemma summarizes all properties we need concerning the boundary term $\mathfrak{b}$ defined in (2.1).

Lemma 2.7 Let $m>0$. There exists a constant $c=c(m)$ such that for every $u, a \geq 0$ we have

(i) $\frac{1}{c}\left|u^{\frac{m+1}{2}}-a^{\frac{m+1}{2}}\right|^{2} \leq \mathfrak{b}\left[u^{m}, a^{m}\right] \leq c\left|u^{\frac{m+1}{2}}-a^{\frac{m+1}{2}}\right|^{2}$.

(ii) $\frac{1}{c}\left|u^{m}-a^{m}\right|^{2} \leq(u+a)^{m-1} \mathfrak{b}\left[u^{m}, a^{m}\right] \leq c\left|u^{m}-a^{m}\right|^{2}$.

Proof The proof of (i) can be found in [4, Lemma 2.3] for $m \geq 1$ and in [3, Lemma 3.4] for $0<m<1$. The inequalities in (ii) are a consequence of (i) and Lemma 2.6.

The following iteration lemma is a well known result and can be found for instance in [8, Chap. I.4, Lemma 4.1].

Lemma 2.8 Let $\left(Y_{i}\right)_{i \in \mathbb{N}_{0}}$ be a sequence of non-negative numbers satisfying

$$
Y_{i+1} \leq \kappa b^{i} Y_{i}^{1+\gamma} \quad \text { for all } i \in \mathbb{N}_{0}
$$

with some positive constants $\kappa, \gamma$ and $b>1$. If

$$
Y_{0} \leq \kappa^{-\frac{1}{\gamma}} b^{-\frac{1}{\gamma^{2}}}
$$

then $Y_{i} \rightarrow 0$ as $i \rightarrow \infty$.

Finally, we recall a parabolic version of the Gagliardo-Nirenberg inequality, see [8, Chapter I, Proposition 3.1] or [2, Lemma 3.1].

Lemma 2.9 Let $Q_{\varrho, \theta}^{-}\left(z_{o}\right) \subset \mathbb{R}^{n+1}$ be a parabolic cylinder and $1<p, r<\infty$. For every

$$
u \in L^{\infty}\left(t_{o}-\theta, t_{o} ; L^{r}\left(B_{\varrho}\left(x_{o}\right)\right)\right) \cap L^{p}\left(t_{o}-\theta, t_{o}, W^{1, p}\left(B_{\varrho}\left(x_{o}\right)\right)\right)
$$

we have $u \in L^{q}\left(Q_{\varrho, \theta}^{-}\left(z_{o}\right)\right)$ for $q=p\left(1+\frac{r}{n}\right)$ with the estimate

$$
\begin{aligned}
& \iint_{Q_{\varrho, \theta}^{-}\left(z_{o}\right)}|u|^{q} \mathrm{~d} x \mathrm{~d} t \\
& \quad \leq c\left(\sup _{t \in\left(t_{o}-\theta, t_{o}\right)} \int_{B_{\varrho}\left(x_{o}\right) \times\{t\}}|u|^{r} \mathrm{~d} x\right)^{\frac{p}{n}} \int_{Q_{\varrho, \theta}^{-}\left(z_{o}\right)}\left[|D u|^{p}+\left|\frac{u}{\varrho}\right|^{p}\right] \mathrm{d} x \mathrm{~d} t,
\end{aligned}
$$

where $c=c(n, p, r)$.

\section{Caccioppoli inequalities}

In this section we derive energy estimates that are crucial in the course of the paper. We start with the energy estimates for weak super-solutions. 
Lemma 3.1 Let $m>0, p>1$ with $m(p-1)>1$ and $u$ be a non-negative weak supersolution to (1.3) in the sense of Definition 1.1, where the vector-field A fulfills the growth and ellipticity assumptions (1.4). Then, there exists a constant $c=c(p, v, L)$ such that on any cylinder $Q_{\varrho, \theta}^{-}\left(z_{o}\right) \Subset \Omega_{T}$ with $\varrho, \theta>0$, and for any $0<r<\varrho, 0<s<\theta$ and $a \geq 0$ the following energy estimates

$$
\begin{aligned}
& \sup _{t \in \Lambda_{s}^{-}\left(t_{o}\right)} \int_{\{u<a\} \cap B_{r}\left(x_{o}\right) \times\{t\}} \mathfrak{b}\left[u^{m}, a^{m}\right] \mathrm{d} x+\iint_{\{u<a\} \cap Q_{r, s}^{-}\left(z_{o}\right)}\left|D u^{m}\right|^{p} \mathrm{~d} x \mathrm{~d} t \\
& \leq c \iint_{\{u<a\} \cap Q_{\varrho, \theta}^{-}\left(z_{o}\right)}\left[\frac{\mathfrak{b}\left[u^{m}, a^{m}\right]}{\theta-s}+\frac{\left|u^{m}-a^{m}\right|^{p}}{(\varrho-r)^{p}}+|F|^{\frac{p}{p-1}}\right] \mathrm{d} x \mathrm{~d} t
\end{aligned}
$$

and

$$
\begin{aligned}
& \sup _{t \in \Lambda_{s}^{-}\left(t_{o}\right)} \int_{\{u<a\} \cap B_{r}\left(x_{o}\right) \times\{t\}} \mathfrak{b}\left[u^{m}, a^{m}\right] \mathrm{d} x \leq \int_{\{u<a\} \cap B_{\varrho}\left(x_{o}\right) \times\left\{t_{o}-s\right\}} \mathfrak{b}\left[u^{m}, a^{m}\right] \mathrm{d} x \\
& +c \iint_{\{u<a\} \cap Q_{\varrho, \theta}^{-}\left(z_{o}\right)}\left[\frac{\left|u^{m}-a^{m}\right|^{p}}{(\varrho-r)^{p}}+|F|^{\frac{p}{p-1}}\right] \mathrm{d} x \mathrm{~d} t
\end{aligned}
$$

hold true, where $\mathfrak{b}[\cdot, \cdot]$ is defined in (2.1).

Proof Throughout the proof we abbreviate $Q_{\varrho, \theta}^{-} \equiv Q_{\varrho, \theta}^{-}\left(z_{o}\right)$ and $B_{\varrho} \equiv B_{\varrho}\left(x_{o}\right)$. Since the claimed estimates are local in nature, we may assume without loss of generality that $u \in C^{0}\left([0, T) ; L^{m+1}(\Omega)\right)$. An approximation argument shows that the mollified weak formulation (2.4) extends to non-negative testing functions $\varphi \in L^{p}\left(0, T ; W_{0}^{1, p}(\Omega)\right) \cap L^{\frac{m+1}{m}}\left(\Omega_{T}\right)$ with compact support, since $\llbracket u \rrbracket_{h} \in C^{0}\left([0, T) ; L^{m+1}(\Omega)\right), \llbracket \mathbf{A}\left(x, t, u, D u^{m}\right) \rrbracket_{h}, \llbracket F \rrbracket_{h} \in$ $L^{\frac{p}{p-1}}\left(\Omega_{T}\right)$ and $u(0) \in L^{m+1}(\Omega)$ by the assumptions on $u$, growth condition (1.4) and Lemma 2.1. We therefore find that

$$
\begin{aligned}
& \iint_{\Omega_{T}}\left[\partial_{t} \llbracket u \rrbracket_{h} \varphi+\llbracket \mathbf{A}\left(x, t, u, D u^{m}\right) \rrbracket_{h} \cdot D \varphi\right] \mathrm{d} x \mathrm{~d} t \\
& \quad \geq \iint_{\Omega_{T}} \llbracket F \rrbracket_{h} \cdot D \varphi \mathrm{d} x \mathrm{~d} t+\frac{1}{h} \int_{\Omega} u(0) \int_{0}^{T} e^{-\frac{s}{h}} \varphi \mathrm{d} s \mathrm{~d} x
\end{aligned}
$$

holds true for any $\varphi \in L^{p}\left(0, T ; W_{0}^{1, p}\left(\Omega, \mathbb{R}_{\geq 0}\right)\right) \cap L^{\frac{m+1}{m}}\left(\Omega_{T}\right)$ with compact support. For $\varepsilon>0$ and $t_{1} \in \Lambda_{s}\left(t_{o}\right)=\left(t_{o}-s, t_{o}\right)$ we define cutoff functions $\eta \in W^{1, \infty}\left(B_{\varrho}\left(x_{o}\right),[0,1]\right)$, $\zeta \in W^{1, \infty}\left(\Lambda_{\theta}\left(t_{o}\right),[0,1]\right)$ and $\psi_{\varepsilon} \in W^{1, \infty}\left(\Lambda_{\theta}\left(t_{o}\right),[0,1]\right)$ which satisfy

$$
\begin{aligned}
& \eta(x)=\left\{\begin{array}{ll}
1, & \text { for } x \in B_{r}\left(x_{o}\right), \\
0, & \text { for } x \in \Omega \backslash B_{\varrho}\left(x_{o}\right),
\end{array} \quad \text { and }|D \eta| \leq \frac{2}{\varrho-r},\right. \\
& \zeta(t)= \begin{cases}1, & \text { for } t \in\left(t_{o}-s, t_{o}+\theta\right), \\
\frac{t-t_{o}+\theta}{\theta-s}, & \text { for } t \in\left(t_{o}-\theta, t_{o}-s\right),\end{cases} \\
& \psi_{\varepsilon}(t)= \begin{cases}1, & \text { for } t \in\left(t_{o}-\theta, t_{1}\right], \\
1-\frac{1}{\varepsilon}\left(t-t_{1}\right), & \text { for } t \in\left(t_{1}, t_{1}+\varepsilon\right), \\
0, & \text { for } t \in\left[t_{1}+\varepsilon, t_{o}\right) .\end{cases}
\end{aligned}
$$

We choose

$$
\varphi(x, t)=\eta^{p}(x) \zeta(t) \psi_{\varepsilon}(t)\left(u^{m}(x, t)-a^{m}\right)_{-}
$$


as testing function in the mollified version (3.3) of the differential equation. For the first term on the left hand side we have

$$
\begin{aligned}
& \iint_{\Omega_{T}} \partial_{t} \llbracket u \rrbracket_{h} \varphi \mathrm{d} x \mathrm{~d} t \\
& =-\iint_{\{u<a\} \cap Q_{\varrho, \theta}^{-}} \eta^{p} \zeta \psi_{\varepsilon} \partial_{t} \llbracket u \rrbracket_{h}\left(\llbracket u \rrbracket_{h}^{m}-a^{m}\right) \mathrm{d} x \mathrm{~d} t \\
& \quad-\iint_{\{u<a\} \cap Q_{\varrho, \theta}^{-}} \eta^{p} \zeta \psi_{\varepsilon} \partial_{t} \llbracket u \rrbracket_{h}\left(u^{m}-\llbracket u \rrbracket_{h}^{m}\right) \mathrm{d} x \mathrm{~d} t \\
& \leq-\iint_{\{u<a\} \cap Q_{\varrho, \theta}^{-}} \eta^{p} \zeta \psi_{\varepsilon} \partial_{t} \llbracket u \rrbracket_{h}\left(\llbracket u \rrbracket_{h}^{m}-a^{m}\right) \mathrm{d} x \mathrm{~d} t \\
& =-\iint_{\{u<a\} \cap Q_{\varrho, \theta}^{-}} \eta^{p} \zeta \psi_{\varepsilon} \partial_{t}\left(\frac{1}{m+1} \llbracket u \rrbracket_{h}^{m+1}+\frac{m}{m+1} a^{m+1}-a^{m} \llbracket u \rrbracket_{h}\right) \mathrm{d} x \mathrm{~d} t \\
& =-\iint_{\{u<a\} \cap Q_{\varrho, \theta}^{-}} \eta^{p} \zeta \psi_{\varepsilon} \partial_{t} \mathfrak{b}\left[\llbracket u \rrbracket_{h}^{m}, a^{m}\right] \mathrm{d} x \mathrm{~d} t \\
& =\iint_{\{u<a\} \cap Q_{\varrho, \theta}^{-}} \eta^{p}\left(\zeta \psi_{\varepsilon}^{\prime}+\psi_{\varepsilon} \zeta^{\prime}\right) \mathfrak{b}\left[\llbracket u \rrbracket_{h}^{m}, a^{m}\right] \mathrm{d} x \mathrm{~d} t,
\end{aligned}
$$

where we used in turn (2.3), the fact that $\left(u-\llbracket u \rrbracket_{h}\right)\left(u^{m}-\llbracket u \rrbracket_{h}^{m}\right) \geq 0$ by monotonicity of $s \mapsto s^{m}$ and the definition of $\mathfrak{b}$. Since $\llbracket u \rrbracket_{h} \rightarrow u$ in $L_{\text {loc }}^{m+1}\left(\Omega_{T}\right)$ in the limit $h \downarrow 0$, we get

$$
\begin{aligned}
& \underset{h \downarrow 0}{\lim \sup } \iint_{\Omega_{T}} \partial_{t} \llbracket u \rrbracket_{h} \varphi \mathrm{d} x \mathrm{~d} t \\
& \quad \leq \iint_{\{u<a\} \cap Q_{\varrho, \theta}^{-}} \eta^{p}\left(\zeta \psi_{\varepsilon}^{\prime}+\psi_{\varepsilon} \zeta^{\prime}\right) \mathfrak{b}\left[u^{m}, a^{m}\right] \mathrm{d} x \mathrm{~d} t=: \mathrm{I}_{\varepsilon}+\mathrm{II}_{\varepsilon},
\end{aligned}
$$

where the meaning of $\mathrm{I}_{\varepsilon}$ and $\mathrm{II}_{\varepsilon}$ is clear in this context. We let $h \downarrow 0$ also in the diffusion term. For the resulting integral we use assumptions (1.4) and Young's inequality to obtain

$$
\begin{aligned}
& \lim _{h \downarrow 0} \iint_{\Omega_{T}} \llbracket \mathbf{A}\left(x, t, u, D u^{m}\right) \rrbracket_{h} \cdot D \varphi \mathrm{d} x \mathrm{~d} t \\
& \quad=\iint_{\Omega_{T}} \mathbf{A}\left(x, t, u, D u^{m}\right) \cdot D \varphi \mathrm{d} x \mathrm{~d} t \\
& \quad=-\iint_{\{u<a\} \cap Q_{\varrho, \theta}^{-}} \mathbf{A}\left(x, t, u, D u^{m}\right) \cdot D\left(\eta^{p} \zeta \psi_{\varepsilon}\left(u^{m}-a^{m}\right)\right) \mathrm{d} x \mathrm{~d} t \\
& \quad=-\iint_{\{u<a\} \cap Q_{\varrho, \theta}^{-}} \mathbf{A}\left(x, t, u, D u^{m}\right) \cdot\left[\eta^{p} \zeta \psi_{\varepsilon} D u^{m}+p \eta^{p-1} \zeta \psi_{\varepsilon}\left(u^{m}-a^{m}\right) D \eta\right] \mathrm{d} x \mathrm{~d} t \\
& \quad \leq \iint_{\{u<a\} \cap Q_{\varrho, \theta}^{-}}\left[-v \eta^{p} \zeta \psi_{\varepsilon}\left|D u^{m}\right|^{p}+p L \eta^{p-1} \zeta \psi_{\varepsilon}\left|D \eta \| u^{m}-a^{m}\right|\left|D u^{m}\right|^{p-1}\right] \mathrm{d} x \mathrm{~d} t \\
& \quad \leq-\frac{\nu}{2} \iint_{\{u<a\} \cap Q_{\varrho, \theta}^{-}} \eta^{p} \zeta \psi_{\varepsilon}\left|D u^{m}\right|^{p} \mathrm{~d} x \mathrm{~d} t+c \iint_{\{u<a\} \cap Q_{\varrho, \theta}^{-}} \frac{\left|u^{m}-a^{m}\right|^{p}}{(\varrho-r)^{p}} \mathrm{~d} x \mathrm{~d} t,
\end{aligned}
$$

where $c=c(p, v, L)$. The second term on the right hand side of (3.3) vanishes in the limit $h \downarrow 0$, since $\varphi(0) \equiv 0$. In the first integral we pass to the limit $h \downarrow 0$ and then apply Young's 
inequality. This yields

$$
\begin{aligned}
& \lim _{h \downarrow 0} \iint_{\Omega_{T}} \llbracket F \rrbracket_{h} \cdot D \varphi \mathrm{d} x \mathrm{~d} t \\
& \geq-\int_{\{u<a\} \cap Q_{Q, \theta}^{-}}\left[\eta^{p} \zeta \psi_{\varepsilon}|F|\left|D u^{m}\right|+|F|\left|u^{m}-a^{m}\right||D \eta|\right] \mathrm{d} x \mathrm{~d} t \\
& \geq-\int_{\{u<a\} \cap Q_{\varrho, \theta}^{-}}\left[\frac{v}{4} \eta^{p} \zeta \psi_{\varepsilon}\left|D u^{m}\right|^{p}+\frac{\left|u^{m}-a^{m}\right|^{p}}{(\varrho-r)^{p}}+c(p, v)|F|^{\frac{p}{p-1}}\right] \mathrm{d} x \mathrm{~d} t .
\end{aligned}
$$

Inserting the preceding estimates into (3.3), we conclude that

$$
\begin{aligned}
& -\mathrm{I}_{\varepsilon}+\frac{v}{4} \iint_{\{u<a\} \cap Q_{\varrho, \theta}^{-}} \eta^{p} \zeta \psi_{\varepsilon}\left|D u^{m}\right|^{p} \mathrm{~d} x \mathrm{~d} t \\
& \quad \leq \mathrm{II}_{\varepsilon}+c(p, v, L) \iint_{\{u<a\} \cap Q_{\varrho, \theta}^{-}}\left[\frac{\left|u^{m}-a^{m}\right|^{p}}{(\varrho-r)^{p}}+|F|^{\frac{p}{p-1}}\right] \mathrm{d} x \mathrm{~d} t .
\end{aligned}
$$

Now, we pass to the limit $\varepsilon \downarrow 0$ in the preceding inequality. Since $u \in C^{0}\left([0, T] ; L^{m+1}(\Omega)\right)$, for any $t_{1} \in \Lambda_{S}\left(t_{o}\right)$ we obtain

$$
\begin{aligned}
\lim _{\varepsilon \downarrow 0}\left(-\mathrm{I}_{\varepsilon}\right) & =\lim _{\varepsilon \downarrow 0} \int_{t_{1}}^{t_{1}+\varepsilon} \int_{\{u<a\} \cap B_{\varrho} \times\{t\}} \eta^{p} \mathfrak{b}\left[u^{m}, a^{m}\right] \mathrm{d} x \mathrm{~d} t \\
& =\int_{\{u<a\} \cap B_{\varrho} \times\left\{t_{1}\right\}} \eta^{p} \mathfrak{b}\left[u^{m}, a^{m}\right] \mathrm{d} x \\
& \geq \int_{\{u<a\} \cap B_{r} \times\left\{t_{1}\right\}} \mathfrak{b}\left[u^{m}, a^{m}\right] \mathrm{d} x .
\end{aligned}
$$

Further, we have

$$
\lim _{\varepsilon \downarrow 0} \iint_{\{u<a\} \cap Q_{Q, \theta}^{-}} \eta^{p} \zeta \psi_{\varepsilon}\left|D u^{m}\right|^{p} \mathrm{~d} x \mathrm{~d} t \geq \iint_{\{u<a\} \cap B_{r} \times\left(t_{o}-s, t_{1}\right)}\left|D u^{m}\right|^{p} \mathrm{~d} x \mathrm{~d} t
$$

and

$$
\mathrm{II}_{\varepsilon} \leq \iint_{\{u<a\} \cap Q_{\varrho, \theta}^{-}} \frac{\mathfrak{b}\left[u^{m}, a^{m}\right]}{\theta-s} \mathrm{~d} x \mathrm{~d} t
$$

Altogether, we deduce the estimate

$$
\begin{gathered}
\int_{\{u<a\} \cap B_{r} \times\left\{t_{1}\right\}} \mathfrak{b}\left[u^{m}, a^{m}\right] \mathrm{d} x+\frac{v}{4} \iint_{\{u<a\} \cap B_{r} \times\left(t_{o}-s, t_{1}\right)}\left|D u^{m}\right|^{p} \mathrm{~d} x \mathrm{~d} t \\
\leq c(p, v, L) \iint_{\{u<a\} \cap Q_{\varrho, \theta}^{-}}\left[\frac{\left|u^{m}-a^{m}\right|^{p}}{(\varrho-r)^{p}}+\frac{\mathfrak{b}\left[u^{m}, a^{m}\right]}{\theta-s}+|F|^{\frac{p}{p-1}}\right] \mathrm{d} x \mathrm{~d} t
\end{gathered}
$$

for any $t_{1} \in \Lambda_{S}\left(t_{o}\right)$. Finally, taking the supremum over $t_{1} \in \Lambda_{S}\left(t_{o}\right)$ in the first term and passing to the limit $t_{1} \uparrow t_{o}$ in the second term yields inequality (3.1). 
In order to prove (3.2) we choose $\varphi(x, t)=\eta^{p}(x) \psi_{\varepsilon}(t)\left(u^{m}(x, t)-a^{m}\right)_{-}$as testing function in (3.3), where $\eta$ is defined as before and

$$
\psi_{\varepsilon}(t)= \begin{cases}0, & \text { for } t \in\left(t_{o}-\theta, t_{1}-\varepsilon\right], \\ \frac{t-t_{1}+\varepsilon}{\varepsilon}, & \text { for } t \in\left(t_{1}-\varepsilon, t_{1}\right), \\ 1, & \text { for } t \in\left[t_{1}, t_{2}\right], \\ \frac{t_{2}-t+\varepsilon}{\varepsilon}, & \text { for } t \in\left(t_{2}, t_{2}+\varepsilon\right), \\ 0, & \text { for } t \in\left[t_{2}+\varepsilon, t_{o}\right),\end{cases}
$$

for $t_{o}-s \leq t_{1}<t_{2}<t_{o}$ and $\varepsilon>0$ small enough. The term involving the time derivative of $\llbracket u \rrbracket_{h}$ is treated as in (3.4). Thus, we find that

$$
\begin{aligned}
& \lim _{\varepsilon \downarrow 0}\left[\limsup _{h \downarrow 0} \iint_{\Omega_{T}} \partial_{t} \llbracket u \rrbracket_{h} \varphi \mathrm{d} x \mathrm{~d} t\right] \\
& \quad \leq \lim _{\varepsilon \downarrow 0} \iint_{\{u<a\} \cap Q_{Q, \theta}^{-}} \eta^{p} \psi_{\varepsilon}^{\prime} \mathfrak{b}\left[u^{m}, a^{m}\right] \mathrm{d} x \mathrm{~d} t \\
& \quad=\int_{\{u<a\} \cap B_{\varrho} \times\left\{t_{1}\right\}} \eta^{p} \mathfrak{b}\left[u^{m}, a^{m}\right] \mathrm{d} x-\int_{\{u<a\} \cap B_{\varrho} \times\left\{t_{2}\right\}} \eta^{p} \mathfrak{b}\left[u^{m}, a^{m}\right] \mathrm{d} x \\
& \quad \leq \int_{\{u<a\} \cap B_{\varrho} \times\left\{t_{1}\right\}} \mathfrak{b}\left[u^{m}, a^{m}\right] \mathrm{d} x-\int_{\{u<a\} \cap B_{r} \times\left\{t_{2}\right\}} \mathfrak{b}\left[u^{m}, a^{m}\right] \mathrm{d} x
\end{aligned}
$$

for any $t_{o}-s \leq t_{1}<t_{2}<t_{o}$. For the diffusion term and the right side the same arguments as in the proof of (3.1) are applicable. Therefore by passing to the limits $h \downarrow 0$ and $\varepsilon \downarrow 0$ we obtain

$$
\begin{aligned}
& \int_{\{u<a\} \cap B_{r} \times\left\{t_{2}\right\}} \mathfrak{b}\left[u^{m}, a^{m}\right] \mathrm{d} x+\frac{v}{2} \iint_{\{u<a\} \cap B_{r} \times\left(t_{1}, t_{2}\right)}\left|D u^{m}\right|^{p} \mathrm{~d} x \mathrm{~d} t \\
& \leq \int_{\{u<a\} \cap B_{Q} \times\left\{t_{1}\right\}} \mathfrak{b}\left[u^{m}, a^{m}\right] \mathrm{d} x \\
& \quad+c(p, v, L) \iint_{\{u<a\} \cap Q_{\varrho, \theta}^{-}}\left[\frac{\left|u^{m}-a^{m}\right|^{p}}{(\varrho-r)^{p}}+|F|^{\frac{p}{p-1}}\right] \mathrm{d} x \mathrm{~d} t
\end{aligned}
$$

for any $t_{o}-s \leq t_{1}<t_{2}<t_{o}$. Omitting the second term on the left side, choosing $t_{1}=t_{o}-s$ and taking the supremum over $t_{2} \in \Lambda_{s}\left(t_{o}\right)$ leads to (3.2).

Similarly, we obtain energy estimates for sub-solutions. However, in the course of the paper we only need the analogue of (3.1).

Lemma 3.2 Under the assumptions of Lemma 3.1 we obtain for any non-negative weak subsolution to (1.3) the energy estimate

$$
\begin{aligned}
& \sup _{t \in \Lambda_{s}^{-}\left(t_{o}\right)} \int_{\{u>a\} \cap B_{r}\left(x_{o}\right) \times\{t\}} \mathfrak{b}\left[u^{m}, a^{m}\right] \mathrm{d} x+\iint_{\{u>a\} \cap Q_{r, s}^{-}\left(z_{o}\right)}\left|D u^{m}\right|^{p} \mathrm{~d} x \mathrm{~d} t \\
& \leq c \iint_{\{u>a\} \cap Q_{\varrho, \theta}^{-}\left(z_{o}\right)}\left[\frac{\mathfrak{b}\left[u^{m}, a^{m}\right]}{\theta-s}+\frac{\left|u^{m}-a^{m}\right|^{p}}{(\varrho-r)^{p}}+|F|^{\frac{p}{p-1}}\right] \mathrm{d} x \mathrm{~d} t,
\end{aligned}
$$

for a constant $c=c(p, v, L)$. 
Proof The proof is analogous to the one of the energy estimate (3.1). Here, we choose the testing function

$$
\varphi(x, t)=\eta^{p}(x) \zeta(t) \psi_{\varepsilon}(t)\left(u^{m}(x, t)-a^{m}\right)_{+}
$$

with the positive part of $u^{m}-a^{m}$ instead of the negative one. Similar arguments as in the proof of (3.1) then lead us to inequality (3.5).

\section{Local boundedness of non-negative weak sub-solutions}

In this section we establish that non-negative weak sub-solutions to (1.3) are locally bounded. We argue by a parabolic version of De Giorgi classes.

Theorem 4.1 Let $m>0$ and $p>1$ with $m(p-1)>1$. Assume that $u$ is a non-negative weak sub-solution to (1.3) in the sense of Definition 1.1 and $F \in L^{\sigma}\left(\Omega_{T}\right)$ with $\sigma>\frac{n+p}{p-1}$. Then $u$ is locally bounded in $\Omega_{T}$ and for any cylinder $Q_{0}:=Q_{\varrho, \theta}^{-}\left(z_{o}\right) \Subset \Omega_{T}$ with $0<\varrho, \theta \leq 1$ the quantitative estimate

$$
\sup _{\frac{1}{2} Q_{0}} u \leq c\left(\frac{1}{\varrho^{p}}+\frac{1}{\theta}\right)^{\frac{n+p}{p(m+1)}}\left[\|u\|_{L^{m p}\left(Q_{0}\right)}^{\frac{m p}{m+1}}+\|F\|_{L^{\sigma}\left(Q_{0}\right)}^{\frac{\sigma}{m+1}}+1\right]
$$

holds true, where $\frac{1}{2} Q_{0}:=Q_{\frac{\rho}{2}, \frac{\theta}{2}}^{-}\left(z_{o}\right)$ and $c$ is a constant depending on $n, m, p, v, L$ and $\sigma$.

Proof Let $m^{\prime}:=\frac{m+1}{m}$ denote the conjugate Hölder exponent of $m+1$. For $i \in \mathbb{N}_{0}$ we define radii $\varrho_{i}$ and times $\theta_{i}$ by

$$
\varrho_{i}:=\frac{1}{2}\left(1+2^{-i}\right) \varrho \quad \text { and } \quad \theta_{i}:=\frac{1}{2}\left(1+2^{-i}\right) \theta .
$$

Throughout the proof, we use the short-hand notation

$$
Q_{i}:=Q_{\varrho_{i}, \tau_{i}}^{-}\left(z_{o}\right) \subset Q_{0} .
$$

Furthermore, for a quantity $k \geq 1$ to be chosen later on, we consider levels

$$
k_{i}:=\left(1-2^{-i}\right)^{\frac{1}{m}} k
$$

and the sequence of integrals

$$
Y_{i}:=\iint_{Q_{i}}\left(u^{m}-k_{i}^{m}\right)_{+}^{p} \mathrm{~d} x \mathrm{~d} t .
$$

Since $u^{m} \in L^{p}\left(\Omega_{T}\right)$ by definition, $Y_{i}$ is finite for any $i \in \mathbb{N}_{0}$. The idea of proof is to show a recursive estimate for $Y_{i}$. To this aim we first use Hölder's inequality to obtain

$$
\begin{aligned}
Y_{i+1} & \leq\left(\iint_{Q_{i+1}}\left(u^{m}-k_{i+1}^{m}\right)_{+}^{\frac{p\left(n+m^{\prime}\right)}{n}} \mathrm{~d} x \mathrm{~d} t\right)^{\frac{n}{n+m^{\prime}}}\left|\left\{u>k_{i+1}\right\} \cap Q_{i+1}\right|^{1-\frac{n}{n+m^{\prime}}} \\
& =: \mathrm{I}^{\frac{n}{n+m^{\prime}}} \cdot\left|\left\{u>k_{i+1}\right\} \cap Q_{i+1}\right|^{1-\frac{n}{n+m^{\prime}}}
\end{aligned}
$$

where the definition of I is clear in this context. First, by the Gagliardo-Nirenberg inequality from Lemma 2.9 we infer

$$
\mathrm{I} \leq c\left[\sup _{t \in\left(t_{o}-\theta_{i+1}, t_{o}\right)} \int_{B_{Q_{i+1}}\left(x_{o}\right) \times\{t\}}\left(u^{m}-k_{i+1}^{m}\right)_{+}^{\frac{m+1}{m}} \mathrm{~d} x\right]^{\frac{p}{n}}
$$




$$
\iint_{Q_{i+1}}\left[\left|D\left(u^{m}-k_{i+1}^{m}\right)_{+}\right|^{p}+\frac{\left(u^{m}-k_{i+1}^{m}\right)_{+}^{p}}{\varrho^{p}}\right] \mathrm{d} x \mathrm{~d} t,
$$

for a constant $c=c(n, m, p)$. We now consider the integrand in the first integral on the right-hand side. For $u \geq k_{i+1}$ we have with the abbreviation

$$
\tilde{k}_{i}^{m}:=\frac{1}{2}\left(k_{i}^{m}+k_{i+1}^{m}\right)<k_{i+1}
$$

that

$$
u^{m}+\tilde{k}_{i}^{m} \leq 2 u^{m} \leq \frac{2 k_{i+1}^{m}}{k_{i+1}^{m}-\tilde{k}_{i}^{m}}\left(u^{m}-\tilde{k}_{i}^{m}\right) \leq 2^{i+3}\left(u^{m}-\tilde{k}_{i}^{m}\right)_{+}
$$

and

$$
u^{m}+\tilde{k}_{i}^{m} \geq u^{m}-\tilde{k}_{i}^{m} .
$$

Therefore, in view of Lemma 2.7 (ii) we obtain

$$
\begin{aligned}
\left(u^{m}-k_{i+1}^{m}\right)_{+}^{\frac{m+1}{m}} & =\left(u^{m}+\tilde{k}_{i}^{m}\right)_{+}^{\frac{1-m}{m}}\left(u^{m}+\tilde{k}_{i}^{m}\right)_{+}^{\frac{m-1}{m}}\left(u^{m}-k_{i+1}^{m}\right)_{+}^{\frac{m+1}{m}} \\
& \leq c 2^{\frac{(m-1)_{+}}{m} i}\left(u+\tilde{k}_{i}\right)_{+}^{1-m}\left(u^{m}-\tilde{k}_{i}^{m}\right)_{+}^{\frac{m-1}{m}}\left(u^{m}-k_{i+1}^{m}\right)_{+}^{\frac{m+1}{m}} \\
& \leq c 2^{\frac{(m-1)_{+}}{m} i}\left(u+\tilde{k}_{i}\right)_{+}^{1-m}\left(u^{m}-\tilde{k}_{i}^{m}\right)_{+}^{2} \\
& \leq c 2^{\frac{(m-1)_{+}}{m} i} \mathfrak{b}\left[u^{m}, \tilde{k}_{i}^{m}\right] \chi_{\left\{u>\tilde{k}_{i}\right\}}
\end{aligned}
$$

Using this inequality above and applying the Caccioppoli inequality (3.5) from Lemma 3.2, yields

$$
\begin{aligned}
\mathrm{I} \leq c & \left.\sup _{t \in\left(t_{o}-\theta_{i+1}, t_{o}\right)} \int_{\left\{u>\tilde{k}_{i}\right\} \cap B_{\varrho_{i+1}}\left(x_{o}\right) \times\{t\}} \mathfrak{b}\left[u^{m}, \tilde{k}_{i}^{m}\right] \mathrm{d} x\right]^{\frac{p}{n}} \\
& \cdot \iint_{\left\{u>\tilde{k}_{i}\right\} \cap Q_{i+1}}\left[\left|D u^{m}\right|^{p}+\frac{\left(u^{m}-\tilde{k}_{i}^{m}\right)^{p}}{\varrho^{p}}\right] \mathrm{d} x \mathrm{~d} t \\
\leq c & {\left[\iint_{\left\{u>\tilde{k}_{i}\right\} \cap Q_{i}}\left[\frac{2^{\frac{(m-1)_{+}}{m}} i \mathfrak{b}\left[u^{m}, \tilde{k}_{i}^{m}\right]}{\theta_{i}-\theta_{i+1}}+\frac{\left(u^{m}-\tilde{k}_{i}^{m}\right)^{p}}{\left(\varrho_{i}-\varrho_{i+1}\right)^{p}}+|F|^{\frac{p}{p-1}}\right] \mathrm{d} x \mathrm{~d} t\right]^{\frac{n+p}{n}}, }
\end{aligned}
$$

for a constant $c=c(n, m, p, v, L)$. For $u>\tilde{k}_{i}$ we now estimate the $\mathfrak{b}$-term with the help of Lemma 2.7 (i), the assumption $m+1 \leq m p$ and the fact that $k_{i}<\tilde{k}_{i}<k$ with $k \geq 1$. In this way we obtain

$$
\begin{aligned}
\mathfrak{b}\left[u^{m}, \tilde{k}_{i}^{m}\right] & \leq\left|u^{\frac{m+1}{2}}-\tilde{k}_{i}^{\frac{m+1}{2}}\right|^{2} \leq 2 u^{m+1} \leq 2\left(u^{m p}+1\right) \\
& \leq c\left[\left(u^{m}-\tilde{k}_{i}^{m}\right)^{p}+k^{m p}\right]=c\left[\left(u^{m}-\tilde{k}_{i}^{m}\right)^{p}+2^{(i+2) p}\left(\tilde{k}_{i}^{m}-k_{i}^{m}\right)^{p}\right] \\
& \leq c 2^{i p}\left(u^{m}-k_{i}^{m}\right)^{p},
\end{aligned}
$$


with $c=c(p)$, so that

$$
\begin{aligned}
\mathrm{I} & \leq c\left[\iint_{\left\{u>\tilde{k}_{i}\right\} \cap Q_{i}}\left[2^{i\left(p+\frac{(m-1)_{+}}{m}\right)}\left(\frac{1}{\varrho^{p}}+\frac{1}{\theta}\right)\left(u^{m}-k_{i}^{m}\right)^{p}+|F|^{\frac{p}{p-1}}\right] \mathrm{d} x \mathrm{~d} t\right]^{\frac{n+p}{n}} \\
& \leq c\left[2^{i\left(p+\frac{(m-1)_{+}}{m}\right)}\left(\frac{1}{\varrho^{p}}+\frac{1}{\theta}\right) Y_{i}+\|F\|_{L^{\sigma}\left(Q_{0}\right)}^{\frac{p}{p-1}}\left|\left\{u>\tilde{k}_{i}\right\} \cap Q_{i}\right|^{1-\frac{p}{\sigma(p-1)}}\right]^{\frac{n+p}{n}},
\end{aligned}
$$

where $c=c(n, m, p, v, L)$. Further, we have that

$$
\left|\left\{u>\tilde{k}_{i}\right\} \cap Q_{i}\right|\left(\tilde{k}_{i}^{m}-k_{i}^{m}\right)_{+}^{p} \leq \iint_{\left\{u>\tilde{k}_{i}\right\} \cap Q_{i}}\left(u^{m}-k_{i}^{m}\right)_{+}^{p} \mathrm{~d} x \mathrm{~d} t \leq Y_{i},
$$

which together with $k \geq 1$ implies that

$$
\left|\left\{u>\tilde{k}_{i}\right\} \cap Q_{i}\right| \leq \frac{2^{(i+2) p}}{k^{m p}} Y_{i} \leq 2^{(i+2) p} Y_{i} .
$$

Finally, the preceding computations together with $0<\varrho \leq 1$ and $Y_{i} \leq\|u\|_{L^{m p}\left(Q_{0}\right)}^{m p}$ lead to

$$
\mathrm{I} \leq c\left[2^{i\left(p+\frac{(m-1)+}{m}\right)}\left(\frac{1}{\varrho^{p}}+\frac{1}{\theta}\right)\left(\|u\|_{L^{m p}\left(Q_{0}\right)}^{\frac{m p^{2}}{\sigma(p-1)}}+\|F\|_{L^{\sigma}\left(Q_{0}\right)}^{\frac{p}{p-1}}\right) Y_{i}^{1-\frac{p}{\sigma(p-1)}}\right]^{\frac{n+p}{n}},
$$

with a constant $c=c(n, m, p, v, L)$. Inserting this inequality into (4.1) and using (4.2), we conclude that

$$
\begin{aligned}
Y_{i+1} \leq & c\left[2^{i\left(p+\frac{(m-1)_{+}}{m}\right)}\left(\frac{1}{\varrho^{p}}+\frac{1}{\theta}\right)\left(\|u\|_{L^{m p}\left(Q_{0}\right)}^{\frac{m p}{\sigma}}+\|F\|_{L^{\sigma}\left(Q_{0}\right)}\right)^{\frac{p}{p-1}} Y_{i}^{1-\frac{p}{\sigma(p-1)}}\right]^{\frac{n+p}{n+m^{\prime}}} \\
& \cdot\left[\frac{2^{i p}}{k^{m p}} Y_{i}\right]^{\frac{m^{\prime}}{n+m^{\prime}}} \\
\leq & \kappa b^{i} Y_{i}^{1+\gamma}
\end{aligned}
$$

where we used the abbreviations

$$
\begin{aligned}
\kappa & :=\frac{c\left(\frac{1}{\varrho^{p}}+\frac{1}{\theta}\right)^{\frac{n+p}{n+m^{\prime}}}}{k^{\frac{p(m+1)}{n+m^{\prime}}}}\left(\|u\|_{L^{m p}\left(Q_{0}\right)}^{\frac{m p}{\sigma}}+\|F\|_{L^{\sigma}\left(Q_{0}\right)}\right)^{\frac{p(n+p)}{(p-1)\left(n+m^{\prime}\right)}}, \\
b & :=2^{\frac{\left(p+\frac{(m-1)+}{m}\right)(n+p)+p m^{\prime}}{n+m^{\prime}}}, \\
\gamma & :=\frac{p}{n+m^{\prime}}\left(1-\frac{n+p}{\sigma(p-1)}\right) .
\end{aligned}
$$

Since $\sigma>\frac{n+p}{p-1}$, we have that $\gamma>0$. Choosing $k \geq 1$ large enough, such that

$$
k \geq c\left(\frac{1}{\varrho^{p}}+\frac{1}{\theta}\right)^{\frac{n+p}{p(m+1)}}\|u\|_{L^{m p}\left(Q_{0}\right)}^{\frac{m p}{m+1}\left(1-\frac{n+p}{\sigma(p-1)}\right)}\left(\|u\|_{L^{m p}\left(Q_{0}\right)}^{\frac{m p}{\sigma}}+\|F\|_{L^{\sigma}\left(Q_{0}\right)}\right)^{\frac{n+p}{(p-1)(m+1)}}
$$

with a suitable constant $c=c(n, m, p, v, L, \sigma)$, we find that

$$
Y_{0}=\iint_{Q_{0}} u^{m p} \mathrm{~d} x \mathrm{~d} t \leq \kappa^{-\frac{1}{\gamma}} b^{-\frac{1}{\gamma^{2}}}
$$

Thus, the assumptions of Lemma 2.8 are satisfied. Consequently we find that $Y_{i} \rightarrow 0$ as $i \rightarrow \infty$, which implies $u \leq k$ a.e. in $\frac{1}{2} Q_{0}$. The claim of the theorem now follows by an application of Young's inequality. 


\section{De Giorgi type lemmas}

In this section we will prove certain De Giorgi type lemmata for weak sub- and supersolutions. We start with the one for super-solutions.

Lemma 5.1 Let $m>0, p>1$ with $m(p-1)>1$ and $u$ be a bounded non-negative weak super-solution to (1.3) in the sense of Definition 1.1, where the vector-field $\mathbf{A}$ satisfies (1.4) and $F \in L^{\sigma}\left(\Omega_{T}\right)$ for some $\sigma>\frac{n+p}{p-1}$. Moreover, consider $z_{o} \in \Omega_{T}$ and $\varrho, \theta, M>0$ such that

$$
Q_{2 \varrho, 2^{p} \tau}^{-}\left(z_{o}\right) \Subset \Omega_{T}, \quad \text { where } \tau:=\theta M^{-d} \varrho^{p} .
$$

Then, there exists $v_{1} \in(0,1)$ depending only on $n, m, p, v, L, \sigma$ and $\theta$, such that: If

$$
\begin{gathered}
\left|\{u<M\} \cap Q_{2 \varrho, 2^{p} \tau}^{-}\left(z_{o}\right)\right| \leq v_{1}\left|Q_{2 \varrho, 2^{p} \tau}^{-}\left(z_{o}\right)\right| \\
\|F\|_{L^{\sigma}\left(\Omega_{T}\right)} \leq\left(\frac{M^{m}}{\varrho}\right)^{p-1}\left|Q_{\varrho, \tau}^{-}\left(z_{o}\right)\right|^{\frac{1}{\sigma}}
\end{gathered}
$$

then

$$
u \geq \frac{M}{2} \quad \text { a.e. in } Q_{\varrho, \tau}^{-}\left(z_{o}\right)
$$

holds true.

Proof For $i \in \mathbb{N}_{0}$ define radii $\varrho_{i}$ and times $\tau_{i}$ by

$$
\varrho_{i}:=\varrho\left(1+2^{-i}\right) \quad \text { and } \quad \tau_{i}:=\theta M^{-d} \varrho_{i}^{p}
$$

as well as levels

$$
k_{i}:=\frac{M}{2}\left(1+2^{-i}\right) .
$$

To shorten notation, we introduce

$$
Q_{i}:=Q_{\varrho_{i}, \tau_{i}}^{-}\left(z_{o}\right), \quad A_{i}:=\left\{u<k_{i}\right\} \cap Q_{i} \quad \text { and } \quad Y_{i}:=\frac{\left|A_{i}\right|}{\left|Q_{i}\right|} \leq 1 .
$$

At this stage, we use the Caccioppoli inequality (3.1). Since $0 \leq u<k_{i}$ on $A_{i}$ and $\frac{M}{2} \leq$ $k_{i} \leq M$ and by Lemma 2.7 (ii), we estimate the term involving $\mathfrak{b}$ on the left-hand side by

$$
\mathfrak{b}\left[u^{m}, k_{i}^{m}\right] \geq \frac{1}{c(m)}\left(k_{i}+u\right)^{1-m}\left(u^{m}-k_{i}^{m}\right)_{-}^{2} \geq \frac{1}{c(m)} M^{1-m}\left(u^{m}-k_{i}^{m}\right)_{-}^{2},
$$

while for the one on the right-hand side we obtain by Lemma 2.7 (i) that

$$
\mathfrak{b}\left[u^{m}, k_{i}^{m}\right] \leq c(m)\left(u^{\frac{m+1}{2}}-k_{i}^{\frac{m+1}{2}}\right)_{-}^{2} .
$$

Thus, we conclude that

$$
\begin{aligned}
& \sup _{t \in\left(t_{o}-\tau_{i+1}, t_{o}\right)} \int_{B_{\varrho_{i+1} \times\{t\}}} M^{1-m}\left(u^{m}-k_{i}^{m}\right)_{-}^{2} \mathrm{~d} x+\iint_{Q_{i+1}}\left|D\left(u^{m}-k_{i}^{m}\right)_{-}\right|^{p} \mathrm{~d} x \mathrm{~d} t \\
& \leq c \iint_{A_{i}}\left[2^{(i+1) p} \frac{\left(u^{m}-k_{i}^{m}\right)_{-}^{p}}{\varrho^{p}}+2^{i p} M^{d} \frac{\left(u^{\frac{m+1}{2}}-k_{i}^{\frac{m+1}{2}}\right)_{-}^{2}}{\theta \varrho^{p}}+|F|^{\frac{p}{p-1}}\right] \mathrm{d} x \mathrm{~d} t
\end{aligned}
$$




$$
\begin{aligned}
& \leq c 2^{i p}\left[\frac{M^{m p}}{\varrho^{p}}\left(1+\frac{1}{\theta}\right)\left|A_{i}\right|^{\frac{p}{\sigma(p-1)}}+\|F\|_{L^{\sigma}\left(\Omega_{T}\right)}^{\frac{p}{p-1}}\right]\left|A_{i}\right|^{1-\frac{p}{\sigma(p-1)}} \\
& \leq \frac{c 2^{i p} M^{m p}}{\varrho^{p}}\left[\left(1+\frac{1}{\theta}\right)\left|A_{i}\right|^{\frac{p}{\sigma(p-1)}}+\left|Q_{i}\right|^{\frac{p}{\sigma(p-1)}}\right]\left|A_{i}\right|^{1-\frac{p}{\sigma(p-1)}} \\
& \leq \frac{c 2^{i p} M^{m p}}{\varrho^{p}}\left|Q_{i}\right|^{\frac{p}{\sigma(p-1)}}\left|A_{i}\right|^{1-\frac{p}{\sigma(p-1)}}
\end{aligned}
$$

where in the second last line we used assumption (5.1). Note that $c=c(m, p, v, L, \theta)$. Next, we use Hölder's inequality with exponents $\frac{n+2}{n}$ and $\frac{n+2}{2}$, the Gagliardo-Nirenberg inequality from Lemma 2.9 with $r=2$ and $p$ and the preceding estimate. This leads to

$$
\begin{aligned}
& \iint_{A_{i+1}}\left(u^{m}-k_{i}^{m}\right)_{-}^{p} \mathrm{~d} x \mathrm{~d} t \\
& \quad \leq\left(\iint_{A_{i+1}}\left(u^{m}-k_{i}^{m}\right)_{-}^{\frac{p(n+2)}{n}} \mathrm{~d} x \mathrm{~d} t\right)^{\frac{n}{n+2}}\left|A_{i}\right|^{\frac{2}{n+2}} \\
& \quad \leq c\left(\sup _{t \in\left(t_{o}-\tau_{i+1}, t_{o}\right)} \int_{B_{Q_{i+1}} \times\{t\}}\left(u^{m}-k_{i}^{m}\right)_{-}^{2} \mathrm{~d} x\right)^{\frac{p}{n+2}} \\
& \quad\left(\iint_{Q_{i+1}}\left|D\left(u^{m}-k_{i}^{m}\right)_{-}\right|^{p}+\frac{\left(u^{m}-k_{i}^{m}\right)_{-}^{p}}{\varrho_{i+1}^{p}} \mathrm{~d} x \mathrm{~d} t\right)^{\frac{n}{n+2}}\left|A_{i}\right|^{\frac{2}{n+2}} \\
& \quad \leq c 2^{i p^{\frac{n+p}{n+2}}} M^{\frac{p(m-1)}{n+2}}\left[\frac{M^{m p}}{\varrho^{p}}\left|Q_{i}\right|^{\frac{p}{\sigma(p-1)}}\left|A_{i}\right|^{-\frac{p}{\sigma(p-1)}}\right]^{\frac{n+p}{n+2}}\left|A_{i}\right|^{1+\frac{p}{n+2}}
\end{aligned}
$$

with a constant $c=c(n, m, p, v, L)$. Moreover, due to Lemma 2.6 we have

$$
\begin{aligned}
\left(k_{i}^{m}-k_{i+1}^{m}\right) & =\left(\frac{M}{2}\right)^{m}\left(\left(1+2^{-i}\right)^{m}-\left(1+2^{-(i+1)}\right)^{m}\right) \\
& \geq \frac{1}{c(m)}\left(\frac{M}{2}\right)^{m}\left(2+2^{-i}+2^{-(i+1)}\right)^{m-1} 2^{-(i+1)} \geq \frac{1}{c(m)} 2^{-i} M^{m},
\end{aligned}
$$

so that

$$
\iint_{A_{i+1}}\left(u^{m}-k_{i}^{m}\right)_{-}^{p} \mathrm{~d} x \mathrm{~d} t \geq\left(k_{i}^{m}-k_{i+1}^{m}\right)^{p}\left|A_{i+1}\right| \geq \frac{1}{c(m, p)} 2^{-i p} M^{m p}\left|A_{i+1}\right| .
$$

Combing the preceding estimates yields

$$
\left|A_{i+1}\right| \leq c 2^{i p\left(1+\frac{n+p}{n+2}\right)} M^{\frac{p(m-1)}{n+2}-m p}\left[\frac{M^{m p}}{\varrho^{p}}\left|Q_{i}\right|^{\frac{p}{\sigma(p-1)}}\left|A_{i}\right|^{-\frac{p}{\sigma(p-1)}}\right]^{\frac{n+p}{n+2}}\left|A_{i}\right|^{1+\frac{p}{n+2}}
$$

with a constant $c=c(n, m, p, v, L, \theta)$. Dividing the above inequality by $\left|Q_{i+1}\right|$, using the fact that $\frac{\left|Q_{i}\right|}{\left|Q_{i+1}\right|}=c(n, p)$ shows that

$$
\begin{aligned}
Y_{i+1} & \leq c 2^{i p\left(1+\frac{n+p}{n+2}\right)} M^{\frac{p(m-1)}{n+2}-m p}\left|Q_{i}\right|^{\frac{p}{n+2}}\left[\frac{M^{m p}}{\varrho^{p}}\right]^{\frac{n+p}{n+2}} Y_{i}^{1+\frac{p}{n+2}-\frac{p(n+p)}{\sigma(n+2)(p-1)}} \\
& \leq c 2^{i p\left(1+\frac{n+p}{n+2}\right)} Y_{i}^{1+\frac{p}{n+2}-\frac{p(n+p)}{\sigma(n+2)(p-1)}}
\end{aligned}
$$

where $c$ depends only on $n, m, p, v, L, \theta$. This brings us into the position to apply Lemma 2.8 with $\kappa=c, b=2^{p\left(m+\frac{n+p}{n+2}\right)}$ and $\gamma=\frac{p}{n+2}-\frac{p(n+p)}{\sigma(n+2)(p-1)}>0$ (since $\sigma>\frac{n+p}{p-1}$ ), where 
$v_{1} \in(0,1)$ can be chosen in dependence on the data. This shows $Y_{i} \rightarrow 0$ as $i \rightarrow \infty$, which yields the claim.

Now we turn our attention to the De Giorgi type lemma for sub-solutions.

Lemma 5.2 Let $m>0, p>1$ with $m(p-1)>1$ and $u$ be a bounded non-negative weak sub-solution to (1.3) in the sense of Definition 1.1, where the vector-field $\mathbf{A}$ satisfies (1.4) and $F \in L^{\sigma}\left(\Omega_{T}\right)$ for some $\sigma>\frac{n+p}{p-1}$. Moreover, consider $z_{o} \in \Omega_{T}, \varrho, \theta, M, \mu_{+}>0$ and $a, \zeta \in(0,1)$, such that

$$
Q_{2 \varrho, 2^{p} \tau}^{-}\left(z_{o}\right) \Subset \Omega_{T}, \quad \text { where } \tau:=\theta M^{-d} \varrho^{p}
$$

and

$$
\sup _{Q_{2 \varrho, 2 p_{\tau}}^{-}\left(z_{o}\right)} u \leq M \leq \mu_{+}
$$

Then, there exists $\nu_{2} \in(0,1)$ depending only on $n, m, p, v, L, \sigma, \theta$, a and $\zeta$ such that: If

$$
\left|\left\{u^{m} \geq \mu_{+}^{m}-\zeta M^{m}\right\} \cap Q_{2 \varrho, 2^{p} \tau}^{-}\left(z_{o}\right)\right| \leq v_{2}\left|Q_{2 \varrho, 2^{p} \tau}^{-}\left(z_{o}\right)\right|
$$

and

$$
\|F\|_{L^{\sigma}\left(\Omega_{T}\right)} \leq\left(\frac{M^{m}}{\varrho}\right)^{p-1}\left|Q_{\varrho, \tau}^{-}\left(z_{o}\right)\right|^{\frac{1}{\sigma}}
$$

then

$$
u^{m} \leq \mu_{+}^{m}-a \zeta M^{m} \quad \text { a.e. in } Q_{\varrho, \tau}^{-}\left(z_{o}\right) .
$$

Proof As before, we define for $i \in \mathbb{N}_{0}$

$$
\varrho_{i}:=\varrho\left(1+2^{-i}\right) \quad \text { and } \quad \tau_{i}:=\theta M^{-d} \varrho_{i}^{p}
$$

as well as levels

$$
k_{i}^{m}:=\mu_{+}^{m}-\left(\frac{1-a}{2^{i}}+a\right) \zeta M^{m}
$$

and sets

$$
Q_{i}:=Q_{\varrho_{i}, \tau_{i}}^{-}\left(z_{o}\right) \quad \text { and } \quad A_{i}:=\left\{u>k_{i}\right\} \cap Q_{i} .
$$

In the following we will apply the Caccioppoli inequality (3.5) from Lemma 3.2. Using the definition of $A_{i}$ and Lemma 2.7 (i), (ii) and the fact that $\frac{1}{c(\zeta)} M \leq k_{i} \leq u \leq M$ on $A_{i}$, we estimate the terms involving $\mathfrak{b}$ by

$$
\begin{aligned}
\frac{1}{c(m, \zeta)} M^{1-m}\left(u^{m}-k_{i}^{m}\right)_{+}^{2} & \leq\left(k_{i}+u\right)^{1-m}\left(u^{m}-k_{i}^{m}\right)_{+}^{2} \\
& \leq c(m) \mathfrak{b}\left[u^{m}, k_{i}^{m}\right] \\
& \leq c(m)\left(u^{\frac{m+1}{2}}-k_{i}^{\frac{m+1}{2}}\right)_{+}^{2} .
\end{aligned}
$$

Thus, by the Caccioppoli inequality (3.5) and assumption (5.4), we obtain

$$
\sup _{t \in\left(t_{o}-\tau_{i+1}, t_{o}\right)} \int_{B_{Q_{i+1}} \times\{t\}} M^{1-m}\left(u^{m}-k_{i}^{m}\right)_{+}^{2} \mathrm{~d} x+\iint_{Q_{i+1}}\left|D\left(u^{m}-k_{i}^{m}\right)_{+}\right|^{p} \mathrm{~d} x \mathrm{~d} t
$$




$$
\begin{aligned}
& \leq c \iint_{A_{i}}\left[2^{(i+1) p} \frac{\left(u^{m}-k_{i}^{m}\right)_{+}^{p}}{\varrho^{p}}+2^{i p} M^{d} \frac{\left(u^{\frac{m+1}{2}}-k_{i}^{\frac{m+1}{2}}\right)_{+}^{2}}{\theta \varrho^{p}}+|F|^{\frac{p}{p-1}}\right] \mathrm{d} x \mathrm{~d} t \\
& \leq c 2^{i p}\left[\frac{M^{m p}}{\varrho^{p}}+\|F\|_{L^{\sigma}\left(\Omega_{T}\right)}^{\frac{p}{p-1}}\left|A_{i}\right|^{-\frac{p}{\sigma(p-1)}}\right]\left|A_{i}\right| \\
& \leq \frac{c 2^{i p} M^{m p}}{\varrho^{p}}\left|Q_{i}\right|^{\frac{p}{\sigma(p-1)}}\left|A_{i}\right|^{1-\frac{p}{\sigma(p-1)}},
\end{aligned}
$$

for a constant $c=c(m, p, v, L, \theta, \zeta)$. Similarly as before, we use Hölder's inequality, the Gagliardo-Nirenberg inequality from Lemma 2.9 with $r=2$ and $p$ and the last estimate to conclude

$$
\begin{aligned}
& \iint_{A_{i+1}}\left(u^{m}-k_{i}^{m}\right)_{+}^{p} \mathrm{~d} x \mathrm{~d} t \\
& \leq\left(\iint_{A_{i+1}}\left(u^{m}-k_{i}^{m}\right)_{+}^{\frac{p(n+2)}{n}} \mathrm{~d} x \mathrm{~d} t\right)^{\frac{n}{n+2}}\left|A_{i}\right|^{\frac{2}{n+2}} \\
& \quad \leq c\left(\sup _{t \in\left(t_{o}-\tau_{i+1}, t_{o}\right)} \int_{B_{\varrho_{i+1}} \times\{t\}}\left(u^{m}-k_{i}^{m}\right)_{+}^{2} \mathrm{~d} x\right)^{\frac{p}{n+2}} \\
& \quad\left(\iint_{Q_{i+1}}\left|D\left(u^{m}-k_{i}^{m}\right)_{+}\right|^{p}+\frac{\left(u^{m}-k_{i}^{m}\right)_{+}^{p}}{\varrho_{i+1}^{p}} \mathrm{~d} x \mathrm{~d} t\right)^{\frac{n}{n+2}}\left|A_{i}\right|^{\frac{2}{n+2}} \\
& \leq c 2^{i p \frac{n+p}{n+2}} M^{\frac{p(m-1)}{n+2}}\left[\frac{M^{m p}}{\varrho^{p}}\left|Q_{i}\right|^{\frac{p}{\sigma(p-1)}}\left|A_{i}\right|^{-\frac{p}{\sigma(p-1)}}\right]^{\frac{n+p}{n+2}}\left|A_{i}\right|^{1+\frac{p}{n+2}}
\end{aligned}
$$

for a constant $c=c(n, m, p, v, L, \theta, \zeta)$. Notice that

$$
\iint_{A_{i+1}}\left(u^{m}-k_{i}^{m}\right)_{+}^{p} \mathrm{~d} x \mathrm{~d} t \geq\left(k_{i+1}^{m}-k_{i}^{m}\right)^{p}\left|A_{i+1}\right|=2^{-(i+1) p}(1-a)^{p} \zeta^{p} M^{m p}\left|A_{i+1}\right| .
$$

Combining the preceding two estimates leads to

$$
\left|A_{i+1}\right| \leq c 2^{i p\left(1+\frac{n+p}{n+2}\right)} M^{\frac{p(m-1)}{n+2}-m p}\left[\frac{M^{m p}}{\varrho^{p}}\left|Q_{i}\right|^{\frac{p}{\sigma(p-1)}}\left|A_{i}\right|^{-\frac{p}{\sigma(p-1)}}\right]^{\frac{n+p}{n+2}}\left|A_{i}\right|^{1+\frac{p}{n+2}},
$$

with a constant $c=c(n, m, p, v, L, \theta, a, \zeta)$. By completely the same reasoning as in the proof of Lemma 5.1 we infer that $Y_{i} \rightarrow 0$ as $i \rightarrow \infty$, provided we choose $\nu_{2} \in(0,1)$ small enough in dependence on the data.

\section{Expansion of positivity}

In this section, we prove the so called Expansion of Positivity of a non-negative weak supersolution $u$. The Expansion of Positivity is crucial in the proof of Harnack's inequality. In a first step we show the following lemma, which ensures a certain propagation of positivity in measure.

Lemma 6.1 Let $m>0, p>1$ with $m(p-1)>1$ and $u$ a non-negative weak supersolution to (1.3), and let $\alpha \in(0,1]$ and $M>0$. Then, there exist $\varepsilon=\varepsilon(m, \alpha) \in(0,1)$ and 
$\delta=\delta(m, p, v, L, \alpha) \in(0,1)$ such that the following holds: Whenever $z_{o}=\left(x_{o}, t_{o}\right) \in \Omega_{T}$ and $\varrho>0$ such that $Q_{\varrho, \delta M^{-d} \varrho^{p}}^{+}\left(z_{o}\right) \subset \Omega_{T}$ and

$$
\left|\left\{u\left(t_{o}\right) \geq M\right\} \cap B_{\varrho}\left(x_{o}\right)\right| \geq \alpha\left|B_{\varrho}\left(x_{o}\right)\right|
$$

and

$$
\|F\|_{L^{\sigma}\left(\Omega_{T}\right)} \leq\left(\frac{M^{m}}{\varrho}\right)^{p-1}\left|Q_{\varrho, \delta M^{-d} \varrho^{p}}^{+}\left(z_{o}\right)\right|^{\frac{1}{\sigma}}
$$

are satisfied, then

$$
\left|\{u(t) \geq \varepsilon M\} \cap B_{\varrho}\left(x_{o}\right)\right| \geq \frac{\alpha}{2}\left|B_{\varrho}\left(x_{o}\right)\right| \text { for all } t \in\left[t_{o}, t_{o}+\delta M^{-d} \varrho^{p}\right) .
$$

Proof In the following we abbreviate $Q_{0}:=Q_{\varrho, \delta M^{-d} \varrho^{p}}^{+}\left(z_{o}\right)$ with $\delta \in(0,1)$ to be chosen later. The idea of the proof is to show that if (6.1) and (6.2) are valid, then

$$
\left|\{u(t)<\varepsilon M\} \cap B_{\varrho}\left(x_{o}\right)\right| \leq\left(1-\frac{\alpha}{2}\right)\left|B_{\varrho}\left(x_{o}\right)\right|
$$

holds true for all $t \in\left[t_{o}, t_{o}+\delta M^{-d} \varrho^{p}\right)$, which is equivalent to (6.3). Therefore in a first step we let $s \in(0,1)$ and compute

$$
\begin{aligned}
& \left|\{u(t)<\varepsilon M\} \cap B_{\varrho}\left(x_{o}\right)\right| \\
& \quad \leq\left|\{u(t)<\varepsilon M\} \cap B_{(1-s) \varrho}\left(x_{o}\right)\right|+\left|B_{\varrho}\left(x_{o}\right) \backslash B_{(1-s) \varrho}\left(x_{o}\right)\right| \\
& \quad \leq\left|\{u(t)<\varepsilon M\} \cap B_{(1-s) \varrho}\left(x_{o}\right)\right|+n s\left|B_{\varrho}\left(x_{o}\right)\right| .
\end{aligned}
$$

To estimate the first term on the right hand side we use the Caccioppoli inequality (3.2) from Lemma 3.1. Taking $r=(1-s) \varrho$ and $a=M$ leads to

$$
\begin{aligned}
& \int_{\{u<M\} \cap B_{(1-s) \varrho}\left(x_{o}\right) \times\{t\}} \mathfrak{b}\left[u^{m}, M^{m}\right] \mathrm{d} x \\
& \quad \leq \int_{\{u<M\} \cap B_{\varrho}\left(x_{o}\right) \times\left\{t_{o}\right\}} \mathfrak{b}\left[u^{m}, M^{m}\right] \mathrm{d} x+c \iint_{\{u<M\} \cap Q_{0}} \frac{\left|u^{m}-M^{m}\right|^{p}}{(s \varrho)^{p}} \mathrm{~d} x \mathrm{~d} t \\
& \quad+c \iint_{\{u<M\} \cap Q_{0}}|F|^{\frac{p}{p-1}} \mathrm{~d} x \mathrm{~d} t \\
& =: \mathrm{I}+\mathrm{II}+\mathrm{III}
\end{aligned}
$$

for any $t \in\left[t_{o}, t_{o}+\delta M^{-d}(1-s)^{p} \varrho^{p}\right)$ with a constant $c=c(p, v, L)$. Recalling the definition of the boundary term $\mathfrak{b}$ from (2.1) we estimate the left hand side by

$$
\begin{aligned}
& \int_{\{u<M\} \cap B_{(1-s) \varrho}\left(x_{o}\right) \times\{t\}} \mathfrak{b}\left[u^{m}, M^{m}\right] \mathrm{d} x \\
& \quad \geq \int_{\{u<\varepsilon M\} \cap B_{(1-s) \varrho}\left(x_{o}\right) \times\{t\}} \mathfrak{b}\left[u^{m}, M^{m}\right] \mathrm{d} x \\
& \quad=\int_{\{u<\varepsilon M\} \cap B_{(1-s) \varrho}\left(x_{o}\right) \times\{t\}} \frac{m}{m+1} M^{m+1}-M^{m} u+\underbrace{\frac{1}{m+1} u^{m+1}}_{\geq 0} \mathrm{~d} x \\
& \quad \geq \int_{\{u<\varepsilon M\} \cap B_{(1-s) \varrho}\left(x_{o}\right) \times\{t\}} \frac{m}{m+1} M^{m+1}-\varepsilon M^{m+1} \mathrm{~d} x \\
& \quad \geq \frac{m}{m+1} M^{m+1}\left(1-\varepsilon \frac{m+1}{m}\right)\left|\{u(t)<\varepsilon M\} \cap B_{(1-s) \varrho}\left(x_{o}\right)\right|
\end{aligned}
$$


for $\varepsilon \in\left(0, \frac{m}{m+1}\right)$ to be chosen later. For the first term on the right-hand side, we use again the definition of $\mathfrak{b}$ and assumption (6.1) to obtain

$$
\begin{aligned}
\mathrm{I} & =\int_{\{u<M\} \cap B_{\varrho}\left(x_{o}\right) \times\left\{t_{o}\right\}} \frac{m}{m+1} M^{m+1} \underbrace{-M^{m} u+\frac{1}{m+1} u^{m+1}}_{\leq 0} \mathrm{~d} x \\
& \leq \frac{m}{m+1} M^{m+1}\left|\left\{u\left(t_{o}\right)<M\right\} \cap B_{\varrho}\left(x_{o}\right)\right| \\
& \leq \frac{m}{m+1} M^{m+1}(1-\alpha)\left|B_{\varrho}\left(x_{o}\right)\right| .
\end{aligned}
$$

Further, we have that

$$
\mathrm{II} \leq c M^{m p}(s \varrho)^{-p}\left|Q_{0}\right|=c \delta s^{-p} M^{m+1}\left|B_{\varrho}\left(x_{o}\right)\right|
$$

and in view of assumption that (6.2) we obtain

$$
\mathrm{III} \leq c\|F\|_{L^{\sigma}\left(\Omega_{T}\right)}^{\frac{p}{p-1}}\left|Q_{0}\right|^{1-\frac{p}{\sigma(p-1)}} \leq c\left(\frac{M^{m}}{\varrho}\right)^{p}\left|Q_{0}\right|=c \delta M^{m+1}\left|B_{\varrho}\left(x_{o}\right)\right| .
$$

Altogether this leads to

$$
\begin{aligned}
& \frac{m}{m+1} M^{m+1}\left(1-\varepsilon \frac{m+1}{m}\right)\left|\{u(t)<\varepsilon M\} \cap B_{(1-s) \varrho}\left(x_{o}\right)\right| \\
& \quad \leq\left[\frac{m}{m+1} M^{m+1}(1-\alpha)+c M^{m+1} \delta\left(s^{-p}+1\right)\right]\left|B_{\varrho}\left(x_{o}\right)\right|,
\end{aligned}
$$

which is the same as

$$
\left|\{u(t)<\varepsilon M\} \cap B_{(1-s) \varrho}\left(x_{o}\right)\right| \leq \frac{1}{1-\varepsilon \frac{m+1}{m}}\left[1-\alpha+c \delta \frac{m+1}{m}\left(s^{-p}+1\right)\right]\left|B_{\varrho}\left(x_{o}\right)\right| .
$$

Combining the last estimate with (6.4), and taking into account that $0<1-\varepsilon \frac{m+1}{m}<1$, we get

$$
\left|\{u(t)<\varepsilon M\} \cap B_{\varrho}\left(x_{o}\right)\right| \leq \frac{1}{1-\varepsilon \frac{m+1}{m}}\left[1-\alpha+c \delta \frac{m+1}{m}\left(s^{-p}+1\right)+n s\right]\left|B_{\varrho}\left(x_{o}\right)\right|
$$

for any $t \in\left[t_{o}, t_{o}+\delta M^{-d} \varrho^{p}\right)$ with $c=c(p, v, L)$. Now we choose $s=\frac{\alpha}{8 n} \in(0,1)$ and thereafter $\delta=\delta(m, p, v, L, \alpha)$ small enough to ensure $c \delta \frac{m+1}{m}\left(s^{-p}+1\right) \leq \frac{\alpha}{8}$. This leads to

$$
\left|\{u(t)<\varepsilon M\} \cap B_{\varrho}\left(x_{o}\right)\right| \leq \frac{1}{1-\varepsilon \frac{m+1}{m}}\left(1-\frac{3 \alpha}{4}\right)\left|B_{\varrho}\left(x_{o}\right)\right|
$$

for all $t \in\left[t_{o}, t_{o}+\delta M^{-d} \varrho^{p}\right)$. Choosing

$$
\varepsilon \leq \frac{m}{m+1}\left(1-\frac{1-\frac{3 \alpha}{4}}{1-\frac{\alpha}{2}}\right) \in(0,1)
$$

we conclude the proof.

Remark 6.2 From the proof of Lemma 6.1 we observe that $\varepsilon$ and $\delta$ are monotonically increasing with respect to $\alpha$.

The preceding lemma at hand, we are now able to prove the Expansion of Positivity for non-negative weak super-solutions to the doubly degenerate equation (1.3). 
Proposition 6.3 (Expansion of Positivity) Let $m>0, p>1$ with $m(p-1)>1$ and $u$ be a non-negative weak super-solution to (1.3). For fixed $\alpha \in(0,1]$ there exist constants $b, \kappa \in(0,1)$ and $c \geq 1$ depending only on $n, m, p, v, L, \sigma$ and $\alpha$ such that the following holds true: We consider $z_{o}=\left(x_{o}, t_{o}\right) \in \Omega_{T}, M>0$ and

$$
\varrho \in\left(0, \varrho_{0}\right], \quad \text { where } \varrho_{0}:=\min \left\{\frac{1}{8} \operatorname{dist}\left(x_{o}, \partial \Omega\right),\left[\frac{\left(T-t_{o}\right)(\kappa M)^{d}}{b}\right]^{\frac{1}{p}}\right\} .
$$

Supposed that

$$
\left|\left\{u\left(t_{o}\right) \geq M\right\} \cap B_{\varrho}\left(x_{o}\right)\right| \geq \alpha\left|B_{\varrho}\left(x_{o}\right)\right|
$$

and

$$
\|F\|_{L^{\sigma}\left(\Omega_{T}\right)} \leq \frac{1}{c}\left(\frac{M^{m}}{\varrho}\right)^{p-1}\left|Q_{\varrho, M^{-d} \varrho^{p}}^{+}\left(z_{o}\right)\right|^{\frac{1}{\sigma}},
$$

are satisfied, then we have

$$
u \geq \kappa M \quad \text { a.e. in } B_{2 \varrho}\left(x_{o}\right) \times\left(t_{o}+\frac{1}{2} b(\kappa M)^{-d} \varrho^{p}, t_{o}+b(\kappa M)^{-d} \varrho^{p}\right] .
$$

Proof The proof of Proposition 6.3 is divided into several steps. Throughout the proof we denote by $\varepsilon=\varepsilon(m, \alpha) \in(0,1)$ and $\delta=\delta(m, p, v, L, \alpha) \in(0,1)$ the constants from Lemma 6.1.

\subsection{Application of lemma 6.1}

For $j_{\star} \in \mathbb{N}$ to be chosen later in dependence on $n, m, p, v, L, \sigma$ and $\alpha$ we define

$$
\varrho_{0}:=\min \left\{\frac{1}{8} \operatorname{dist}\left(x_{o}, \partial \Omega\right), \varrho_{1}\right\}, \quad \text { where } \varrho_{1}:=\left[\frac{\left(T-t_{o}\right) M^{d}}{\delta} \exp \left(-\frac{2^{2 p+j_{\star} d}}{\delta \varepsilon^{d}}\right)\right]^{\frac{1}{p}}
$$

and

$$
s_{0}:=\frac{1}{M}\left(\frac{\delta \varrho_{1}^{p}}{T-t_{o}}\right)^{\frac{1}{d}}=\exp \left(-\frac{2^{2 p+j_{\star} d}}{\delta d \varepsilon^{d}}\right)<1
$$

Note that

$$
B_{8 \varrho}\left(x_{o}\right) \times\left[t_{o}, t_{o}+\delta(s M)^{-d} \varrho^{p}\right) \subset \Omega_{T} \quad \text { for all } \varrho \in\left(0, \varrho_{0}\right] \text { and } s \in\left[s_{0}, 1\right] .
$$

Now we fix $\varrho \in\left(0, \varrho_{0}\right]$ and assume that (6.6) is satisfied and that

$$
\|F\|_{L^{\sigma}\left(\Omega_{T}\right)} \leq\left(\frac{\left(s_{0} M\right)^{m}}{\varrho}\right)^{p-1}\left|Q_{\varrho, \delta M^{-d} \varrho^{p}}^{+}\left(z_{o}\right)\right|^{\frac{1}{\sigma}} .
$$

Then, the assumptions of Lemma 6.1 are fulfilled with $M$ replaced by $s M$ for any $s \in\left[s_{0}, 1\right]$. Thus, we find that

$$
\left|\{u(t) \geq \varepsilon s M\} \cap B_{\varrho}\left(x_{o}\right)\right| \geq \frac{\alpha}{2}\left|B_{\varrho}\left(x_{o}\right)\right|
$$

for all $s \in\left[s_{0}, 1\right]$ and all $t \in\left[t_{o}, t_{o}+\delta(s M)^{-d} \varrho^{p}\right)$. 


\subsection{Transforming to another problem}

For $\tau \geq 0$ we let $s(\tau):=e^{-\frac{\tau}{d}}$. Then, we have $s(\tau) \in\left[s_{0}, 1\right]$ for $\tau \in\left[0, \tau_{0}\right]$, where

$$
\tau_{0}:=d \ln \left(\frac{1}{s_{0}}\right)=\frac{2^{2 p+j_{\star} d}}{\delta \varepsilon^{d}} .
$$

Next, we define

$$
\widetilde{\Phi}(\tau):=\delta(s(\tau) M)^{-d} \varrho^{p}=\delta M^{-d} \varrho^{p} e^{\tau} \quad \text { for } \tau \in\left[0, \tau_{0}\right] .
$$

From Step 6.1 we deduce that for any $\tau \in\left[0, \tau_{0}\right]$ there holds

$$
\left[t_{o}, t_{o}+\widetilde{\Phi}(\tau)\right) \subset(0, T)
$$

and

$$
\left|\{u(t) \geq \varepsilon s(\tau) M\} \cap B_{\varrho}\left(x_{o}\right)\right| \geq \frac{\alpha}{2}\left|B_{\varrho}\left(x_{o}\right)\right| \quad \text { for all } t \in\left[t_{o}, t_{o}+\widetilde{\Phi}(\tau)\right) .
$$

In particular, letting

$$
\Phi(\tau):=t_{o}+\widetilde{\Phi}(\tau) \quad \text { for } \tau \in\left[0, \tau_{0}\right]
$$

we have that

$$
\left|\{u(\Phi(\tau)) \geq \varepsilon s(\tau) M\} \cap B_{\varrho}\left(x_{o}\right)\right| \geq \frac{\alpha}{2}\left|B_{\varrho}\left(x_{o}\right)\right| \quad \text { for all } \tau \in\left[0, \tau_{0}\right] .
$$

Finally, we let $\gamma(\tau):=\widetilde{\Phi}(\tau)^{\frac{1}{d}}$. Then, Corollary 2.4 ensures that

$$
v(x, \tau):=\gamma(\tau) u(x, \Phi(\tau))
$$

is a non-negative weak super-solution to

$$
\partial_{t} v-\operatorname{div} \widehat{\mathbf{A}}\left(x, \tau, v, D v^{m}\right)=\operatorname{div} \widehat{F} \quad \text { in } B_{4 \varrho} \times\left(0, \tau_{0}\right)
$$

with

$$
\widehat{\mathbf{A}}(x, \tau, v, \xi):=\gamma(\tau) \Phi^{\prime}(\tau) \mathbf{A}\left(x, \Phi(\tau), \frac{v}{\gamma(\tau)}, \frac{\xi}{\gamma^{m}(\tau)}\right)
$$

and

$$
\widehat{F}(x, \tau):=\gamma(\tau) \Phi^{\prime}(\tau) F(x, \Phi(\tau)) .
$$

Using the growth assumptions (1.4) of $\mathbf{A}$ together with the definition of the functions $\Phi$ and $\gamma$ we compute that $\widehat{\mathbf{A}}$ satisfies the growth and ellipticity conditions

$$
\widehat{\mathbf{A}}(x, \tau, u, \xi) \cdot \xi \geq \frac{v}{2}|\xi|^{p} \quad \text { and } \quad|\widehat{\mathbf{A}}(x, \tau, u, \xi)| \leq \frac{L}{2}|\xi|^{p-1} .
$$

Defining

$$
k_{0}:=\varepsilon\left(\delta \varrho^{p}\right)^{\frac{1}{d}},
$$

we observe that $k_{0}=\varepsilon \gamma(\tau) s(\tau) M$ for any $\tau \in\left[0, \tau_{0}\right]$ and therefore inequality (6.9) can be rewritten as

$$
\left|\left\{v(\tau) \geq k_{0}\right\} \cap B_{\varrho}\left(x_{o}\right)\right| \geq \frac{\alpha}{2}\left|B_{\varrho}\left(x_{o}\right)\right| \quad \text { for all } \tau \in\left[0, \tau_{0}\right] .
$$




\subsection{Gradient estimates on intrinsic sublevel sets}

Next, we define

$$
\vartheta:=\left(\frac{2^{j_{\star}}}{k_{0}}\right)^{d}=\frac{1}{\delta \varrho^{p}}\left(\frac{2^{j_{\star}}}{\varepsilon}\right)^{d}
$$

and consider cylinders

$$
\begin{aligned}
& Q_{r}^{\vartheta}:=Q_{r}^{\vartheta}\left(z_{o}\right)=B_{r}\left(x_{o}\right) \times\left[0, \vartheta r^{p}\right), \\
& \check{Q}_{r}^{\vartheta}:=\breve{Q}_{r}^{\vartheta}\left(z_{o}\right)=B_{r}\left(x_{o}\right) \times\left[0, \vartheta\left(\frac{r}{2}\right)^{p}\right), \\
& \widehat{Q}_{r}^{\vartheta}:=\widehat{Q}_{r}^{\vartheta}\left(z_{o}\right)=B_{r}\left(x_{o}\right) \times\left[\vartheta\left(\frac{r}{2}\right)^{p}, \vartheta r^{p}\right)
\end{aligned}
$$

for $0<r \leq 8 \varrho$. Moreover, for $j=1, \ldots, j_{\star}$, we let

$$
k_{j}:=2^{-j} k_{0}, \quad A_{j}(\tau):=\left\{v(\tau)<k_{j}\right\} \cap B_{4 \varrho}\left(x_{o}\right), \quad A_{j}:=\left\{v<k_{j}\right\} \cap \widehat{Q}_{4 \varrho}^{\vartheta}
$$

and observe that

$$
\left|A_{j}\right|=\int_{\vartheta(2 \varrho)^{p}}^{\vartheta(4 \varrho)^{p}}\left|A_{j}(\tau)\right| \mathrm{d} \tau .
$$

Further, a simple computation shows that

$$
\widehat{Q}_{\varrho}^{\vartheta} \subset \check{Q}_{2 \varrho}^{\vartheta} \quad \text { and } \quad \check{Q}_{8 \varrho}^{\vartheta} \subset B_{8 \varrho} \times\left[0, \tau_{0}\right)
$$

By definition of $\widehat{F}$ and Hölder's inequality, we obtain for $q \in[1, \sigma]$ that

$$
\begin{aligned}
& \iint_{\check{Q}_{8 \varrho}^{\vartheta}}|\widehat{F}|^{q} \mathrm{~d} x \mathrm{~d} \tau=\iint_{\check{Q}_{8 \varrho}^{\vartheta}}\left|\gamma(\tau) \Phi^{\prime}(\tau) F(x, \Phi(\tau))\right|^{q} \mathrm{~d} x \mathrm{~d} \tau \\
& \quad \leq\left\|\gamma(\tau)^{q} \Phi^{\prime}(\tau)^{q\left(1-\frac{1}{\sigma}\right)}\right\|_{L^{\infty}\left(0, \vartheta(4 \varrho)^{p}\right)} \iint_{\check{Q}_{8 \varrho}^{\vartheta}} \Phi^{\prime}(\tau)^{\frac{q}{\sigma}}|F(x, \Phi(\tau))|^{q} \mathrm{~d} x \mathrm{~d} \tau \\
& \quad \leq\left(\delta M^{-d} \varrho^{p} e^{\vartheta(4 \varrho)^{p}}\right)^{q\left(1+\frac{1}{d}-\frac{1}{\sigma}\right)}\left(\iint_{\check{Q}_{8 \varrho}^{\vartheta}} \Phi^{\prime}(\tau)|F(x, \Phi(\tau))|^{\sigma} \mathrm{d} x \mathrm{~d} \tau\right)^{\frac{q}{\sigma}}\left|\check{Q}_{8 \varrho}^{\vartheta}\right|^{1-\frac{q}{\sigma}} \\
& \quad \leq c\left[\left(M^{-d} \varrho^{p}\right)^{1+\frac{1}{d}-\frac{1}{\sigma}}\left|\check{Q}_{8 \varrho}^{\vartheta}\right|^{-\frac{1}{\sigma}}\|F\|_{L^{\sigma}\left(\Omega_{T}\right)}\right]^{q}\left|\check{Q}_{8 \varrho}^{\vartheta}\right|,
\end{aligned}
$$

where in the second last line we used the area formula and the fact that $\vartheta(4 \varrho)^{p}=\frac{4^{p}}{\delta}\left(\frac{2^{j_{\star}}}{\varepsilon}\right)^{d}$, so that $c=c\left(n, m, p, v, L, \sigma, \alpha, j_{\star}\right)$. Assuming that

$$
\|F\|_{L^{\sigma}\left(\Omega_{T}\right)} \leq \frac{1}{c_{\star}}\left(\frac{M^{m}}{\varrho}\right)^{p-1}\left|Q_{\varrho, M^{-d} \varrho^{p}}^{+}\left(z_{o}\right)\right|^{\frac{1}{\sigma}}
$$

for some constant $c_{\star} \geq 1$ to be chosen later, we further estimate

$$
\iint_{\check{Q}_{8 \varrho}^{\vartheta}}|\widehat{F}|^{q} \mathrm{~d} x \mathrm{~d} \tau \leq \frac{c}{c_{\star}^{q}}\left[\varrho^{1+\frac{p}{d}-\frac{p}{\sigma}} \vartheta^{-\frac{1}{\sigma}}\right]^{q}\left|\check{Q}_{8 \varrho}^{\vartheta}\right| \leq \frac{c}{c_{\star}}\left(\frac{k_{j_{\star}}^{m}}{\varrho}\right)^{q(p-1)}\left|\check{Q}_{8 \varrho}^{\vartheta}\right|,
$$

again with a constant $c=c\left(n, m, p, v, L, \sigma, \alpha, j_{\star}\right)$. Therefore, we may choose $c_{\star}$ in dependence on $n, m, p, v, L, \sigma, \alpha$ and $j_{\star}$ in such a way that

$$
\iint_{\check{Q}_{8 \varrho}^{\vartheta}}|\widehat{F}|^{q} \mathrm{~d} x \mathrm{~d} \tau \leq\left(\frac{k_{j_{\star}}^{m}}{2 \varrho}\right)^{q(p-1)}\left|\check{Q}_{4 \varrho}^{\vartheta}\right|
$$


holds true for any $q \in[1, \sigma]$. Note that we replaced $\varrho$ by $2 \varrho$ in the denominator and $\left|\breve{Q}_{8 \varrho}^{\vartheta}\right|$ by $\left|\breve{Q}_{4 \varrho}^{\vartheta}\right|$ for later purpose. We observe that

$$
\widehat{Q}_{4 \varrho}^{\vartheta}=Q_{4 \varrho, \vartheta\left(4 p-2^{p}\right) \varrho^{p}}^{-}\left(x_{o}, \vartheta(4 \varrho)^{p}\right) \quad \text { and } \quad \check{Q}_{8 \varrho}^{\vartheta}=Q_{8 \varrho, \vartheta(4 \varrho)^{p}}^{-}\left(x_{o}, \vartheta(4 \varrho)^{p}\right) .
$$

Thus, the Caccioppoli inequality (3.1) from Lemma 3.1 together with Lemma 2.7 (i), estimate (6.12) with $q=\frac{p}{p-1}$ and the fact that $\left|\breve{Q}_{8 \varrho}^{\vartheta}\right| /\left|\widehat{Q}_{4 \varrho}^{\vartheta}\right|=c(n)$ implies

$$
\begin{aligned}
\iint_{A_{j}}\left|D v^{m}\right|^{p} \mathrm{~d} x \mathrm{~d} \tau & =\iint_{\left\{v<k_{j}\right\} \cap \widehat{Q}_{4 \varrho}^{\vartheta}}\left|D v^{m}\right|^{p} \mathrm{~d} x \mathrm{~d} \tau \\
& \leq c \iint_{\left\{v<k_{j}\right\} \cap \check{Q}_{8 \varrho}^{\vartheta}}\left[\frac{\left|v^{m}-k_{j}^{m}\right|^{p}}{(4 \varrho)^{p}}+\frac{\mathfrak{b}\left[v^{m}, k_{j}^{m}\right]}{\vartheta(2 \varrho)^{p}}+|\widehat{F}|^{\frac{p}{p-1}}\right] \mathrm{d} x \mathrm{~d} \tau \\
& \leq c\left[\frac{k_{j}^{m p}}{(4 \varrho)^{p}}\left|\check{Q}_{8 \varrho}^{\vartheta}\right|+\frac{k_{j}^{m+1}}{\vartheta(4 \varrho)^{p}}\left|\check{Q}_{8 \varrho}^{\vartheta}\right|+\frac{k_{j_{\star}}^{m p}}{\varrho^{p}}\left|\check{Q}_{8 \varrho}^{\vartheta}\right|\right] \\
& \leq c \frac{k_{j}^{m p}}{\varrho^{p}}\left|\widehat{Q}_{4 \varrho}^{\vartheta}\right|
\end{aligned}
$$

with $c=c(n, m, p, v, L)$.

\subsection{Measure estimates for intrinsic sublevel sets}

Now, we exploit the estimate

$$
k_{j}^{m}-k_{j+1}^{m}=\left(2^{-j m} k_{0}^{m}-2^{-(j+1) m} k_{0}^{m}\right)=2^{-j m}\left(1-2^{-m}\right) k_{0}^{m} \geq c(m) k_{j}^{m}
$$

with $c(m) \in(0,1)$ together with Lemma 2.5 and inequality $(6.10)$ to obtain

$$
\begin{aligned}
c(m) k_{j}^{m}\left|A_{j+1}(\tau)\right| & \leq\left(k_{j}^{m}-k_{j+1}^{m}\right)\left|A_{j+1}(\tau)\right| \\
& \leq \frac{c(n) \varrho^{n+1}}{\left|B_{4 \varrho}\left(x_{o}\right) \backslash A_{j}(\tau)\right|} \int_{B_{4 \varrho}\left(x_{o}\right) \cap\left\{k_{j+1}^{m}<v^{m}(\tau)<k_{j}^{m}\right\}}\left|D v^{m}(\tau)\right| \mathrm{d} x \\
& \leq \frac{c(n) \varrho}{\alpha} \int_{A_{j}(\tau) \backslash A_{j+1}(\tau)}\left|D v^{m}(\tau)\right| \mathrm{d} x
\end{aligned}
$$

for all $j=0, \ldots, j_{\star}$ and all $\tau \in\left(0, \vartheta(4 \varrho)^{p}\right)$. We integrate this inequality with respect to $\tau$ over $\left(\vartheta(2 \varrho)^{p}, \vartheta(4 \varrho)^{p}\right)$, apply Hölder's inequality on the right-hand side and use the gradient bound (6.13) to get

$$
\begin{aligned}
k_{j}^{m}\left|A_{j+1}\right| & \leq \frac{c(n, m) \varrho}{\alpha} \int_{A_{j} \backslash A_{j+1}}\left|D v^{m}\right| \mathrm{d} x \mathrm{~d} \tau \\
& \leq \frac{c(n, m) \varrho}{\alpha}\left(\int_{A_{j}}\left|D v^{m}\right|^{p} \mathrm{~d} x \mathrm{~d} \tau\right)^{\frac{1}{p}}\left|A_{j} \backslash A_{j+1}\right|^{\frac{p-1}{p}} \\
& \leq \frac{c(n, m, p, v, L)}{\alpha} k_{j}^{m}\left|A_{j} \backslash A_{j+1}\right|^{\frac{p-1}{p}}\left|\widehat{Q}_{4 \varrho}^{\vartheta}\right|^{\frac{1}{p}}
\end{aligned}
$$


Dividing both sides by $k_{j}^{m}>0$ and summing over $j=0, \ldots, j_{\star}-1$, we find that

$$
j_{\star}\left|A_{j_{\star}}\right|^{\frac{p}{p-1}} \leq \sum_{j=1}^{j_{\star}}\left|A_{j}\right|^{\frac{p}{p-1}} \leq \frac{c}{\alpha^{\frac{p}{p-1}}}\left|\widehat{Q}_{4 \varrho}^{\vartheta}\right|^{\frac{1}{p-1}} \sum_{j=0}^{j_{\star}-1}\left|A_{j} \backslash A_{j+1}\right| \leq \frac{c}{\alpha^{\frac{p}{p-1}}}\left|\widehat{Q}_{4 \varrho}^{\vartheta}\right|^{\frac{p}{p-1}}
$$

so that

$$
\left|\left\{v<k_{j_{\star}}\right\} \cap \widehat{Q}_{4 \varrho}^{\vartheta}\right|=\left|A_{j_{\star}}\right| \leq \alpha^{-1}\left(\frac{c}{j_{\star}}\right)^{\frac{p-1}{p}}\left|\widehat{Q}_{4 \varrho}^{\vartheta}\right|
$$

for a constant $c$ depending only on $n, m, p, v$ and $L$.

\subsection{Application of De Giorgi type lemma 5.1}

At this stage, we exploit Lemma 5.1. Observe that the cylinder $\widehat{Q}_{4 \varrho}^{\vartheta}=Q_{4 \varrho, \vartheta\left(4 p-2^{p}\right) \varrho^{p}}^{-}\left(x_{o}, \vartheta(4 \varrho)^{p}\right)$ satisfies the requirements of the Lemma with $\varrho, \theta$ and $M$ replaced by $2 \varrho, \frac{4^{p}-2^{p}}{4^{p}}$ and $k_{j_{\star}}$. Then, the constant $v_{1}$ from Lemma 5.1 depends only on $n, m, p, v, L$ and $\sigma$, but is independent of $j_{\star}$. Note that (5.1) is implied by (6.12) applied with $q=\sigma$. Thus, choosing $j_{\star}$ large enough, so that

$$
\left(\frac{c}{j_{\star}}\right)^{\frac{p-1}{p}} \leq \alpha v_{1}
$$

all assumptions of Lemma 5.1 are satisfied and we conclude that

$$
v \geq \frac{1}{2} k_{j_{\star}} \quad \text { a.e. in } B_{2 \varrho}\left(x_{o}\right) \times\left(\left(4^{p}-2^{p}+1\right) \vartheta \varrho^{p}, \vartheta(4 \varrho)^{p}\right) .
$$

Note that $j_{\star}$ depends on $n, m, p, v, L, \sigma$ and $\alpha$. This also fixes $c_{\star}$ in (6.11) in dependence on $n, m, p, v, L, \sigma$ and $\alpha$. In turn, we choose $c \geq 1$ in dependence on $n, m, p, v, L, \sigma$ and $\alpha$ in such a way that condition (6.7) implies the validity of (6.11) and (6.8).

\subsection{Returning to the original problem and conclusion}

Finally we use the definition of $v$ and $k_{0}$ to rewrite (6.15) as

$$
u(x, \Phi(\tau)) \geq 2^{-\left(j_{\star}+1\right)} e^{-\frac{\tau}{d}} \varepsilon M \geq \kappa M
$$

for a.e. $(x, \tau) \in B_{2 \varrho} \times\left(\left(4^{p}-2^{p}+1\right) \vartheta \varrho^{p}, \vartheta(4 \varrho)^{p}\right]$, where

$$
\kappa=\kappa(n, m, p, v, L, \alpha):=2^{-\left(j_{\star}+1\right)} \varepsilon e^{-\frac{\vartheta(4 \rho)^{p}}{d}} .
$$

Returning to the original time variable, we obtain

$$
u \geq \kappa M \quad \text { a.e. in } B_{2 \varrho} \times\left(t_{o}+\beta b(\kappa M)^{-d} \varrho^{p}, t_{o}+b(\kappa M)^{-d} \varrho^{p}\right]
$$

with $b:=\delta \varepsilon^{d} 2^{-\left(j_{\star}+1\right) d} \in(0,1)$ and $\beta:=e^{-\left(2^{p}-1\right) \vartheta \varrho^{p}}$ depending only on the data. Note that by the definitions of $b$ and $\kappa$ we have $\frac{1}{\delta} \exp \left(-\frac{2^{2 p+j_{\star} d}}{\delta \varepsilon^{d}}\right)=\frac{\kappa^{d}}{b}$, so that $\varrho_{0}$ can be re-written exactly as in (6.5). Since $\beta \leq \frac{1}{2}$ this completes the proof of Proposition 6.3.

Remark 6.4 From the proof of Proposition 6.3 we observe that

$$
\frac{b}{\kappa^{d}}=\delta \exp \left(\frac{4^{p}}{\delta}\left(\frac{2^{j_{\star}}}{\varepsilon}\right)^{d}\right)>4^{p} \delta \exp \left(\frac{1}{\delta}\right)>4^{p} .
$$


Moreover, the parameter $b$ in Proposition 6.3 is monotonically increasing with respect to $\alpha$. This can be seen from the definition $b=\delta \varepsilon^{d} 2^{-\left(j_{\star}+1\right) d}$, where $j_{\star}$ is decreasing and $\varepsilon$ and $\delta$ are increasing with respect to $\alpha$; see Remark 6.2.

\section{Harnack's inequality}

We are now ready to prove our main result, Theorem 1.2. In the following section, the second (forward in time) inequality of (1.8) is shown. In a subsequent step, we ensure the validity of the first (backward in time) inequality of (1.8).

\subsection{Forward inequality}

Let $c_{o} \geq 1$ to be fixed later, consider $\left(x_{o}, t_{o}\right) \in \Omega_{T}$ with $u\left(x_{o}, t_{o}\right)>0$ and define

$$
\theta=\left(\frac{c_{o}}{u\left(x_{o}, t_{o}\right)}\right)^{d}
$$

Moreover, assume that $\varrho>0$ is small enough so that $B_{9 \varrho}\left(x_{o}\right) \times\left(t_{o}-2 \theta \varrho^{p}, t_{o}+2 \theta \varrho^{p}\right) \Subset \Omega_{T}$. Note that the stronger assumption $B_{9 \varrho}\left(x_{o}\right) \times\left(t_{o}-4 \theta \varrho^{p}, t_{o}+4 \theta \varrho^{p}\right) \Subset \Omega_{T}$ will only be needed in the proof of the backward Harnack inequality. Finally, we define the rescaled function

$$
v(x, t):=\frac{1}{u\left(x_{o}, t_{o}\right)} u(\tilde{x}(x), \tilde{t}(t)) \quad \text { in } B_{9}(0) \times\left(-2 c_{o}^{d}, 2 c_{o}^{d}\right),
$$

where $(\tilde{x}, \tilde{t}): \widehat{\Omega_{T}} \rightarrow \Omega_{T}$ with $\widehat{\Omega_{T}}:=\left\{(x, t) \in \mathbb{R}^{n+1}:(\tilde{x}, \tilde{t}) \in \Omega_{T}\right\}$ is defined by

$$
\tilde{x}(x):=x_{o}+\varrho x \quad \text { and } \quad \tilde{t}(t):=t_{o}+\frac{t \varrho^{p}}{u\left(x_{o}, t_{o}\right)^{d}} .
$$

A straightforward computation shows that $v$ is a bounded, continuous, non-negative weak super-solution of

$$
\partial_{t} v-\operatorname{div} \tilde{\mathbf{A}}\left(x, t, v, D v^{m}\right)=\operatorname{div} \tilde{F}
$$

in $B_{9}(0) \times\left(-2 c_{o}^{d}, 2 c_{o}^{d}\right)$ in the sense of Definition 1.1 with

$$
\tilde{\mathbf{A}}(x, t, v, \zeta)=\frac{\varrho^{p-1}}{u\left(x_{o}, t_{o}\right)^{d+1}} \mathbf{A}\left(\tilde{x}, \tilde{t}, u\left(x_{o}, t_{o}\right) v, \frac{u\left(x_{o}, t_{o}\right)^{m}}{\varrho} \zeta\right)
$$

and

$$
\tilde{F}(x, t)=\frac{\varrho^{p-1}}{u\left(x_{o}, t_{o}\right)^{d+1}} F(\tilde{x}, \tilde{t}) .
$$

The main step towards Theorem 1.2 is the following lemma. After returning to the original variables this proves the intrinsic forward Harnack inequality, i.e. the second inequality of (1.8). Indeed, if Lemma 7.1 is valid, we obtain that

$$
\|F\|_{L^{\sigma}\left(\Omega_{T}\right)} \geq \gamma_{0}\left|B_{1}(0)\right|^{\frac{1}{\sigma}} \varrho^{\frac{n+p}{\sigma}-(p-1)} u\left(x_{o}, t_{o}\right)^{m(p-1)-\frac{d}{\sigma}}
$$


or

$$
u\left(x_{o}, t_{o}\right) \leq \frac{1}{\gamma_{1}} u\left(\cdot, t_{o}+\theta \varrho^{p}\right) \quad \text { in } B_{\varrho}\left(x_{o}\right),
$$

which shows the second inequality of (1.8) for $\gamma=\max \left\{\frac{1}{\gamma_{0}\left|B_{1}(0)\right|^{1 / \sigma}}, \frac{1}{\gamma_{1}}\right\}$.

Lemma 7.1 For $v$, $\tilde{\mathbf{A}}$ and $\tilde{F}$ as above, there exist constants $\gamma_{0}, \gamma_{1} \in(0,1)$ and $c_{o}>1$ depending only on the data, but independent of $u\left(x_{o}, t_{o}\right)$ such that either

$$
\|\tilde{F}\|_{L^{\sigma}\left(\widehat{\Omega_{T}}\right)} \geq \gamma_{0}\left|B_{1}(0)\right|^{\frac{1}{\sigma}}
$$

or

$$
v\left(\cdot, c_{o}^{d}\right) \geq \gamma_{1} \quad \text { in } B_{1}(0)
$$

Proof In the following we abbreviate $Q_{r}^{-}:=Q_{r, r^{p}}^{-}(0)=B_{r}(0) \times\left(-r^{p}, 0\right]$ for $r>0$. For $\tau \in[0,1)$ we consider the family of cylinders $\left\{Q_{\tau}^{-}\right\}$and the functions $M, N:[0,1) \rightarrow$ $[0, \infty)$ defined by

$$
M(\tau):=\sup _{Q_{\tau}^{-}} v, \quad N(\tau):=(1-\tau)^{-\delta},
$$

with $\delta>1$ to be chosen later on. Note that the functions $M$ and $N$ are both monotonically increasing and $M_{0}=1=N_{0}$, since $v(0,0)=1$. Moreover, as $\tau \uparrow 1, N(\tau) \rightarrow \infty$ while $M(\tau)$ remains bounded, since $v$ is bounded in $Q_{1}^{-}$. Together with the continuity of $v$ this ensures that there exist

$$
\tau_{\star}:=\max \{\tau \in[0,1): M(\tau)=N(\tau)\}
$$

and $\left(x_{\star}, t_{\star}\right) \in Q_{\tau_{\star}}^{-}$such that

$$
v\left(x_{\star}, t_{\star}\right)=M\left(\tau_{\star}\right)=N\left(\tau_{\star}\right)=\left(1-\tau_{\star}\right)^{-\delta} .
$$

Let $\tilde{n} \in \mathbb{N}_{\geq 2}$ such that $2^{1-\tilde{n}}<1-\tau_{\star} \leq 2^{2-\tilde{n}}$ and define $r:=2^{-\tilde{n}}$. Then $\tau_{\star}+r<$ $\tau_{\star}+\frac{1}{2}\left(1-\tau_{\star}\right)=\frac{1+\tau_{\star}}{2}$, which implies

$$
\left(x_{\star}, t_{\star}\right)+Q_{r}^{-} \subset Q_{\frac{1+\tau_{\star}}{2}}^{-} \subset Q_{1}^{-} .
$$

Moreover, by definition of $M, N$ and $\tau_{\star}$ we have

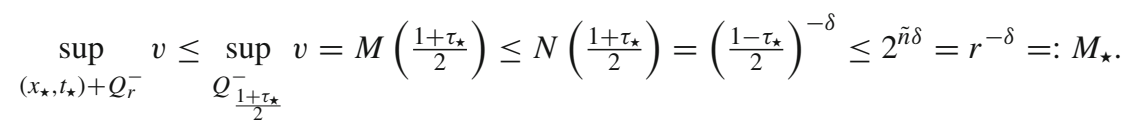

Observe that $M_{\star}>1$. Next, on the cylinder $Q_{r, M_{\star}^{-d} r^{p}}^{-}\left(x_{\star}, t_{\star}\right) \subset\left(x_{\star}, t_{\star}\right)+Q_{r}^{-}$we apply the De Giorgi type Lemma 5.2 to $v$ with

$$
\zeta=1-2^{-4 \delta m}, \quad a=\frac{1-2^{-3 \delta m}}{1-2^{-4 \delta m}}
$$

and $\left(\mu_{+}, M, \theta, \varrho\right)$ replaced by $\left(M_{\star}, M_{\star}, 1, \frac{r}{2}\right)$. Indeed, hypothesis (5.2) is satisfied, since

$$
\left.\sup _{Q_{r, M_{\star}^{-}}^{-} d_{r} p} v \leq \sup _{\left(x_{\star}, t_{\star}\right)} v \leq M_{\star}\right)+Q_{r}^{-}
$$


By $\tilde{v}$ we denote the constant $v_{2}$ from Lemma 5.2 depending on $n, m, p, v, L, \theta, a, \zeta$; hence $\tilde{v}=\tilde{v}(n, m, p, v, L, \delta)$. Moreover, observe that

$$
v^{m}\left(x_{\star}, t_{\star}\right)=\left(1-\tau_{\star}\right)^{-\delta m} \geq 2^{-2 \delta m} r^{-\delta m}=2^{-2 \delta m} M_{\star}^{m}>M_{\star}^{m}-a \zeta M_{\star}^{m} .
$$

This shows that conclusion (5.5) of Lemma 5.2 is false. Hence, either (5.3) or (5.4) is violated. This means, we either have

$$
\|\tilde{F}\|_{L^{\sigma}\left(\widehat{\Omega_{T}}\right)}>\left(\frac{2 M_{\star}^{m}}{r}\right)^{p-1}\left|Q_{r, M_{\star}^{-d} r^{p}}^{-}\left(x_{\star}, t_{\star}\right)\right|^{\frac{1}{\sigma}}
$$

or

$$
\left|\left\{v \geq 2^{-4 \delta} M_{\star}\right\} \cap Q_{r, M_{\star}^{-d} r^{p}}^{-}\left(x_{\star}, t_{\star}\right)\right|>\tilde{v}\left|Q_{r, M_{\star}^{-d} r^{p}}^{-}\left(x_{\star}, t_{\star}\right)\right| .
$$

If (7.4) is satisfied, by Fubini's theorem there exists $\bar{t}_{\star} \in\left(t_{\star}-M_{\star}^{-d} r^{p}, t_{\star}\right]$ with

$$
\left|\left\{v\left(\bar{t}_{\star}\right) \geq 2^{-4 \delta} M_{\star}\right\} \cap B_{r}\left(x_{\star}\right)\right|>\tilde{v}\left|B_{r}\left(x_{\star}\right)\right| .
$$

By $\tilde{b}, \tilde{\kappa} \in(0,1)$ and $\tilde{c} \geq 1$ we denote the constants $b, \kappa, c$ from the Expansion of Positivity in Proposition 6.3 applied with $\alpha=\tilde{v}$. Note that $\tilde{b}, \tilde{\kappa}$ and $\tilde{c}$ depend on $n, m, p, v, L, \sigma$ and $\delta$. Supposed that

$$
r \leq \min \left\{\frac{1}{8} \operatorname{dist}\left(x_{\star}, \partial B_{9}(0)\right),\left[\frac{\left(2 c_{o}^{d}-\bar{t}_{\star}\right)\left(2^{-4 \delta} \tilde{\kappa} M_{\star}\right)^{d}}{\tilde{b}}\right]^{\frac{1}{p}}\right\},
$$

we are allowed to apply Proposition 6.3 with $(F, \alpha, M, \varrho)$ replaced by $\left(\tilde{F}, \tilde{v}, 2^{-4 \delta} M_{\star}, r\right)$ and conclude that either

$$
\|\tilde{F}\|_{L^{\sigma}\left(\widehat{\Omega_{T}}\right)} \geq \frac{1}{\tilde{c}}\left(\frac{\left(2^{-4 \delta} M_{\star}\right)^{m}}{r}\right)^{p-1}\left|Q_{r,\left(2^{-4 \delta} M_{\star}\right)^{-d_{r} p}}^{+}\right|^{\frac{1}{\sigma}}
$$

or

$$
v \geq 2^{-4 \delta} \tilde{\kappa} M_{\star} \text { in } B_{2 r}\left(x_{\star}\right) \times\left(\bar{t}_{\star}+\frac{1}{2} \tilde{b}\left(2^{-4 \delta} \tilde{\kappa} M_{\star}\right)^{-d} r^{p}, \bar{t}_{\star}+\tilde{b}\left(2^{-4 \delta} \tilde{\kappa} M_{\star}\right)^{-d} r^{p}\right]
$$

holds true. In the second case we find that

$$
\left|\left\{v\left(\tilde{t}_{o}\right) \geq 2^{-4 \delta} \tilde{\kappa} M_{\star}\right\} \cap B_{2 r}\left(x_{\star}\right)\right|=\left|B_{2 r}\left(x_{\star}\right)\right|,
$$

where $\tilde{t}_{o}:=\bar{t}_{\star}+\tilde{b}\left(2^{-4 \delta} \tilde{\kappa} M_{\star}\right)^{-d} r^{p}$. This allows to apply the Expansion of Positivity in the next step with $\alpha=1$. Therefore, by $b, \kappa \in(0,1)$ and $c \geq 1$ we denote the constants $b, \kappa, c$ from Proposition 6.3 applied with $\alpha=1$. Then, $b, \kappa$ and $c$ depend on $n, m, p, v, L$ and $\sigma$, but not on $\delta$. Supposed that

$$
2 r \leq \min \left\{\frac{1}{8} \operatorname{dist}\left(x_{\star}, \partial B_{9}(0)\right),\left[\frac{\left(2 c_{o}^{d}-\tilde{t}_{o}\right)\left(2^{-4 \delta} \tilde{\kappa} \kappa M_{\star}\right)^{d}}{b}\right]^{\frac{1}{p}}\right\},
$$

we may apply Proposition 6.3 with $(F, \alpha, M, \varrho)$ replaced by $\left(\tilde{F}, 1,2^{-4 \delta} \tilde{\kappa} M_{\star}, 2 r\right)$ and conclude that either

$$
\|\tilde{F}\|_{L^{\sigma}\left(\widehat{\Omega_{T}}\right)} \geq \frac{1}{c}\left(\frac{\left(2^{-4 \delta} \tilde{\kappa} M_{\star}\right)^{m}}{2 r}\right)^{p-1}\left|Q_{2 r,\left(2^{-4 \delta} \tilde{\kappa} M_{\star}\right)^{-d}(2 r)^{p}}\right|^{\frac{1}{\sigma}}
$$


or

$$
v \geq 2^{-4 \delta} \tilde{\kappa} \kappa M_{\star} \quad \text { in } B_{4 r}\left(x_{\star}\right) \times\left(t_{1}-\frac{1}{2} b\left(2^{-4 \delta} \tilde{\kappa} \kappa M_{\star}\right)^{-d}(2 r)^{p}, t_{1}\right]
$$

holds true, where $t_{1}:=\tilde{t}_{o}+b\left(2^{-4 \delta} \tilde{\kappa} \kappa M_{\star}\right)^{-d}(2 r)^{p}$. In the second case, we have

$$
\left|\left\{v\left(t_{1}\right)>2^{-4 \delta} \tilde{\kappa} \kappa M_{\star}\right\} \cap B_{4 r}\left(x_{\star}\right)\right|=\left|B_{4 r}\left(x_{\star}\right)\right| .
$$

We recursively define $t_{2}, \ldots, t_{\tilde{n}}$ by

$$
t_{j}:=t_{j-1}+b\left(2^{-4 \delta} \tilde{\kappa} \kappa^{j} M_{\star}\right)^{-d}\left(2^{j} r\right)^{p}
$$

for $j \in\{2, \ldots, \tilde{n}\}$. Iterating the procedure of Expansion of Positivity we arrive at the following assertion. Supposed that

$$
2^{j} r \leq \min \left\{\frac{1}{8} \operatorname{dist}\left(x_{\star}, \partial B_{9}(0)\right),\left[\frac{\left(2 c_{o}^{d}-t_{j-1}\right)\left(2^{-4 \delta} \tilde{\kappa} \kappa^{j} M_{\star}\right)^{d}}{b}\right]^{\frac{1}{p}}\right\},
$$

for every $j=2, \ldots, \tilde{n}$, we find that either

$$
\|\tilde{F}\|_{L^{\sigma}\left(\widehat{\left.\Omega_{T}\right)}\right.}>\frac{1}{c}\left(\frac{\left(2^{-4 \delta} \tilde{\kappa} \kappa^{j-1} M_{\star}\right)^{m}}{2^{j} r}\right)^{p-1}\left|Q_{2^{j} r,\left(2^{-4 \delta} \tilde{\kappa} \kappa^{j-1} M_{\star}\right)^{-d}\left(2^{j} r\right)^{p}}^{+}\right|^{\frac{1}{\sigma}}
$$

is satisfied for some $j \in\{2, \ldots, \tilde{n}\}$ or

$$
v \geq 2^{-4 \delta} \tilde{\kappa} \kappa^{\tilde{n}} M_{\star} \text { in } B_{2^{\tilde{n}+1} r}\left(x_{\star}\right) \times\left(t_{\tilde{n}}-\frac{1}{2} b\left(2^{-4 \delta} \tilde{\kappa} \kappa^{\tilde{n}} M_{\star}\right)^{-d}\left(2^{\tilde{n}} r\right)^{p}, t_{\tilde{n}}\right] .
$$

We first ensure that (7.10) is satisfied for $\tilde{n}$. We note that $2^{\tilde{n}} r=1$. Since $x_{\star} \in B_{1}(0)$, we immediately observe that $2^{\tilde{n}} r=1 \leq \frac{1}{8} \operatorname{dist}\left(x_{\star}, \partial B_{9}(0)\right)$. Next, we choose $\delta>1$ in dependence on $n, m, p, v, L$ and $\sigma$ such that $2^{\delta} \kappa=1$, which is possible, since $\kappa$ is independent of $\delta$. In view of the definition of $M_{\star}$ we find that

$$
2^{-4 \delta} \tilde{\kappa} \kappa^{\tilde{n}} M_{\star}=2^{-4 \delta} \tilde{\kappa} \kappa^{\tilde{n}} 2^{\tilde{n} \delta}=2^{-4 \delta} \tilde{\kappa}\left(2^{\delta} \kappa\right)^{\tilde{n}}=2^{-4 \delta} \tilde{\kappa}=: \gamma_{1} \in(0,1) .
$$

Note that $\gamma_{1}$ depends on $n, m, p, v, L$ and $\sigma$. The second condition in (7.10) is equivalent to $t_{\tilde{n}} \leq 2 c_{o}^{d}$. Therefore, we compute

$$
\begin{aligned}
t_{\tilde{n}} & =\bar{t}_{\star}+\tilde{b}\left(2^{-4 \delta} \tilde{\kappa} M_{\star}\right)^{-d} r^{p}+b \sum_{j=1}^{\tilde{n}}\left(2^{-4 \delta} \tilde{\kappa} \kappa^{j} M_{\star}\right)^{-d}\left(2^{j} r\right)^{p} \\
& =\bar{t}_{\star}+\frac{\tilde{b}}{\gamma_{1}^{d}}\left(\frac{\kappa^{d}}{2^{p}}\right)^{\tilde{n}}+\frac{2^{p} b}{\gamma_{1}^{d}\left(2^{p}-\kappa^{d}\right)}\left[1-\left(\frac{\kappa^{d}}{2^{p}}\right)^{\tilde{n}}\right] \\
& =\bar{t}_{\star}+\frac{2^{p} b}{\gamma_{1}^{d}\left(2^{p}-\kappa^{d}\right)}-\frac{1}{\gamma_{1}^{d}}\left[\frac{2^{p} b}{2^{p}-\kappa^{d}}-\tilde{b}\right]\left(\frac{\kappa^{d}}{2^{p}}\right)^{\tilde{n}} .
\end{aligned}
$$

We note that due to Remark 6.4 we have $\tilde{b} \leq b$ and therefore the expression $\frac{2^{p} b}{2^{p}-\kappa^{d}}-\tilde{b}$ is positive. Hence, choosing $c_{o}$ such that

$$
2 c_{o}^{d} \geq \frac{1}{\gamma_{1}^{d}} \frac{2^{p} b}{\left(2^{p}-\kappa^{d}\right)}
$$

and taking into account that $\bar{t}_{\star} \leq 0$ we find that

$$
t_{\tilde{n}} \leq \bar{t}_{\star}+2 c_{o}^{d} \leq 2 c_{o}^{d} .
$$


Provided that (7.13) holds true, (7.10) is satisfied for $\tilde{n}$ and in turn implies that (7.10) is satisfied for any $j=2, \ldots, \tilde{n}$ and in particular also (7.5) and (7.8) are satisfied.

To summarize, we have now shown that either (7.12) is satisfied or one of the alternatives (7.6), (7.9) or (7.11) if $c_{o}$ is chosen large enough. We start with the former case where (7.12) is satisfied. Since $2^{\tilde{n}} r=1$ we have $B_{1}(0) \subset B_{2}\left(x_{\star}\right)=B_{2^{\tilde{n}+1} r}\left(x_{\star}\right)$, so that

$$
v(t) \geq \gamma_{1} \quad \text { in } B_{1}(0) \text { for any } t \in\left(t_{\tilde{n}}-\frac{1}{2} b \gamma_{1}^{-d}, t_{\tilde{n}}\right] .
$$

Unfortunately, the interval depends on $\tilde{n}$ and hence on $v$. Therefore, we need to find a subinterval which is independent of $\tilde{n}$. In view of Remark 6.4 we have $\frac{b}{\tilde{\kappa}^{d}} \geq \frac{\tilde{b}}{\tilde{\kappa}^{d}}>4^{p}$ and hence $\gamma_{1}^{d}=2^{-4 \delta d} \tilde{\kappa}^{d}<2^{-2 p-4 \delta d} b<2^{-2 p} b$. Therefore, we observe from the preceding computation of $t_{\tilde{n}}$ that

$$
\begin{aligned}
t_{\tilde{n}} & \geq \bar{t}_{\star}+\frac{2^{p} b}{\gamma_{1}^{d}\left(2^{p}-\kappa^{d}\right)}\left[1-\left(\frac{\kappa^{d}}{2^{p}}\right)^{\tilde{n}}\right] \geq-1+\frac{2^{p} b}{\gamma_{1}^{d}\left(2^{p}-\kappa^{d}\right)}\left[1-\left(\frac{\kappa^{d}}{2^{p}}\right)^{2}\right] \\
& >\frac{2^{p} b}{\gamma_{1}^{d}\left(2^{p}-\kappa^{d}\right)}-\frac{\kappa^{2 d} b}{2^{p} \gamma_{1}^{d}\left(2^{p}-\kappa^{d}\right)}-\frac{b}{2^{2 p} \gamma_{1}^{d}} .
\end{aligned}
$$

In the second term on the right-hand side we use $\kappa<4^{-p} b<4^{-p}$, which once again is a consequence of Remark 6.4. This leads us to the lower bound

$$
t_{\tilde{n}}>\frac{2^{p} b}{\gamma_{1}^{d}\left(2^{p}-\kappa^{d}\right)}-\frac{b}{2^{3 p} \gamma_{1}^{d}}-\frac{b}{2^{2 p} \gamma_{1}^{d}}>\frac{2^{p} b}{\gamma_{1}^{d}\left(2^{p}-\kappa^{d}\right)}-\frac{b}{2^{2 p-1} \gamma_{1}^{d}} .
$$

For the left interval limit in (7.14) we obtain

$$
t_{\tilde{n}}-\frac{1}{2} b \gamma_{1}^{-d} \leq \frac{2^{p} b}{\gamma_{1}^{d}\left(2^{p}-\kappa^{d}\right)}-\frac{b}{2 \gamma_{1}^{d}} .
$$

The preceding computations show that with the choice

$$
c_{o}^{d}:=\frac{2^{p} b}{\gamma_{1}^{d}\left(2^{p}-\kappa^{d}\right)}-\frac{b}{2^{p} \gamma_{1}^{d}}>1
$$

we have

$$
c_{o}^{d} \in\left(t_{\tilde{n}}-\frac{1}{2} b \gamma_{1}^{-d}, t_{\tilde{n}}\right] .
$$

Note that $c_{o}$ depends on $n, m, p, v, L$ and $\sigma$ and a straightforward calculation shows that (7.13) is satisfied. From (7.14) we now conclude that

$$
v\left(c_{o}^{d}\right) \geq \gamma_{1} \quad \text { in } B_{1}(0),
$$

which concludes the proof of the lemma in the case that (7.12) is satisfied.

Finally, we are left with the case where one of the alternatives (7.6), (7.9) or (7.11) is satisfied. In any of these cases we conclude that

$$
\|\tilde{F}\|_{L^{\sigma}\left(\widehat{\Omega_{T}}\right)} \geq \gamma_{0}\left|B_{1}(0)\right|^{\frac{1}{\sigma}}, \quad \text { where } \gamma_{0}:=\frac{1}{\max \{c, \tilde{c}\}}\left(\frac{\tilde{\kappa}}{2^{4 \delta} \kappa}\right)^{d+1-\frac{d}{\sigma}}
$$

is valid. We note that $\gamma_{0} \in(0,1)$, since $\tilde{\kappa} \leq \kappa$ by Remark 6.4 and that $\gamma_{0}$ depends on $n, m, p, v, L$ and $\sigma$. This concludes the proof of the lemma. 


\subsection{Backward inequality}

With the intrinsic forward Harnack inequality on hand, we are able to show the intrinsic backward Harnack inequality, i.e. the first inequality of (1.8). Actually, in the following we will prove the more general version

$$
\left(c_{1} \gamma^{c_{2}}\right)^{-1} \sup _{B_{\varrho}\left(x_{o}\right)} u\left(\cdot, t_{o}-\left(c_{1} \gamma^{c_{2}-1}\right)^{-d} \theta \varrho^{p}\right) \leq u\left(x_{o}, t_{o}\right)
$$

with positive constants $c_{1}, c_{2}$ such that $c_{1} \gamma^{c_{2}-2}>1$, which implies the first inequality of (1.8) by choosing $c_{1}=c_{2}=2$. We have already fixed $\left(x_{o}, t_{o}\right) \in \Omega_{T}$ with $u\left(x_{o}, t_{o}\right)>0$. Now we assume that $B_{9}\left(x_{o}\right) \times\left(t_{o}-4 \theta \varrho^{p}, t_{o}+4 \theta \varrho^{p}\right) \Subset \Omega_{T}$. Moreover, let $c_{1}, c_{2}>0$ be positive constants such that $c_{1} \gamma^{c_{2}-2}>1$ and suppose that alternative (1.7) is not valid, i.e.

$$
\|F\|_{L^{\sigma}\left(\Omega_{T}\right)} \varrho^{p-1-\frac{n+p}{\sigma}}<\frac{1}{\gamma} u\left(x_{o}, t_{o}\right)^{d+1-\frac{d}{\sigma}} .
$$

In order to prove the backward Harnack inequality, we consider two alternatives. First, we assume that

$$
u\left(x_{o}, t\right)<c_{1} \gamma^{c_{2}-1} u\left(x_{o}, t_{o}\right) \quad \text { for all } t \in\left(t_{o}-2 \theta \varrho^{p}, t_{o}\right)
$$

with $\gamma$ as in the right-hand side of (1.8). Our aim is to prove that (7.18) implies

$$
\sup _{B_{\varrho}\left(x_{o}\right)} u\left(\cdot, t_{o}-\left(c_{1} \gamma^{c_{2}-1}\right)^{-d} \theta \varrho^{p}\right)<c_{1} \gamma^{c_{2}} u\left(x_{o}, t_{o}\right) .
$$

Indeed, assume that (7.19) was not satisfied. Then there exists $x_{\star} \in B_{\varrho}\left(x_{o}\right)$ such that $u\left(x_{\star}, t_{1}\right)=c_{1} \gamma^{c_{2}} u\left(x_{o}, t_{o}\right)$, where we abbreviated $t_{1}:=t_{o}-\left(c_{1} \gamma^{c_{2}-1}\right)^{-d} \theta \varrho^{p}$, since $u$ is continuous and (7.18) is in force. Let $\theta_{\star}:=c_{o}^{d} u\left(x_{\star}, t_{1}\right)^{-d}$. A simple calculation shows that

$$
\left\{\begin{array}{l}
t_{1}-2 \theta_{\star} \varrho^{p}=t_{o}-\left(1+2 \gamma^{-d}\right)\left(c_{1} \gamma^{c_{2}-1}\right)^{-d} \theta \varrho^{p}, \\
t_{1}+2 \theta_{\star} \varrho^{p}=t_{o}-\left(1-2 \gamma^{-d}\right)\left(c_{1} \gamma^{c_{2}-1}\right)^{-d} \theta \varrho^{p} .
\end{array}\right.
$$

Since $d>0, \gamma>1$ and $c_{1} \gamma^{c_{2}-1}>1$, this implies $\left(t_{1}-2 \theta_{\star} \varrho^{p}, t_{1}+2 \theta_{\star} \varrho^{p}\right) \subset\left(t_{o}-\right.$ $\left.4 \theta \varrho^{p}, t_{o}+4 \theta \varrho^{p}\right) \Subset \Omega_{T}$. Thus, we are able to apply the forward Harnack inequality with $\left(x_{o}, t_{o}\right)$ replaced by $\left(x_{\star}, t_{1}\right)$. This leads to

$$
\|F\|_{L^{\sigma}\left(\Omega_{T}\right)} \varrho^{p-1-\frac{n+p}{\sigma}} \geq \frac{1}{\gamma} u\left(x_{\star}, t_{1}\right)^{d+1-\frac{d}{\sigma}}>\frac{1}{\gamma} u\left(x_{o}, t_{o}\right)^{d+1-\frac{d}{\sigma}},
$$

which contradicts (7.17), or

$$
u\left(x_{\star}, t_{1}\right) \leq \gamma \inf _{B_{\varrho}\left(x_{\star}\right)} u\left(\cdot, t_{1}+\theta_{\star} \varrho^{p}\right) .
$$

In view of (7.18) and the facts that $x_{o} \in B_{\varrho}\left(x_{\star}\right)$ and $t_{1}+\theta_{\star} \varrho^{p}<t_{o}$, this yields the contradiction

$$
c_{1} \gamma^{c_{2}} u\left(x_{o}, t_{o}\right)=u\left(x_{\star}, t_{1}\right) \leq \gamma u\left(x_{o}, t_{1}+\theta_{\star} \varrho^{p}\right)<c_{1} \gamma^{c_{2}} u\left(x_{o}, t_{o}\right) .
$$

Therefore (7.18) implies (7.19).

It remains to treat the case where (7.18) is violated. This means that there exists $t \in$ $\left(t_{o}-2 \theta \varrho^{p}, t_{o}\right)$ such that $u\left(x_{o}, t\right)=c_{1} \gamma^{c_{2}-1} u\left(x_{o}, t_{o}\right)$. We define $\tau$ as the largest value with this property (note that $u$ is continuous) and let

$$
\theta_{\tau}:=\left(\frac{c_{o}}{u\left(x_{o}, \tau\right)}\right)^{d}=\left(c_{1} \gamma^{c_{2}-1}\right)^{-d} \theta
$$


We claim that

$$
t_{o}-\tau>\theta_{\tau} Q^{p}
$$

Indeed, if (7.20) was not valid, there existed $0<\tilde{\varrho} \leq \varrho$ such that

$$
t_{o}-\tau=\theta_{\tau} \tilde{\varrho}^{p} .
$$

Computing that $\left(\tau-2 \theta_{\tau} \tilde{\varrho}^{p}, \tau+2 \theta_{\tau} \tilde{\varrho}^{p}\right) \subset\left(t_{o}-4 \theta \varrho^{p}, t_{o}+4 \theta \varrho^{p}\right)$, we are allowed to apply the forward Harnack inequality with $\left(x_{o}, \tau\right)$ instead of $\left(x_{o}, t_{o}\right)$. This gives that either

$$
\|F\|_{L^{\sigma}\left(\Omega_{T}\right)} \tilde{\varrho}^{p-1-\frac{n+p}{\sigma}} \geq \frac{1}{\gamma} u\left(x_{o}, \tau\right)^{d+1-\frac{d}{\sigma}}>\frac{1}{\gamma} u\left(x_{o}, t_{o}\right)^{d+1-\frac{d}{\sigma}},
$$

or

$$
c_{1} \gamma^{c_{2}-1} u\left(x_{o}, t_{o}\right)=u\left(x_{o}, \tau\right) \leq \gamma u\left(x_{o}, \tau+\theta_{\tau} \tilde{\varrho}^{p}\right)=\gamma u\left(x_{o}, t_{o}\right) .
$$

holds true. The former one contradicts (7.17), while the latter one contradicts $c_{1} \gamma^{c_{2}-2}>1$. Therefore, (7.20) is valid. Next, we define

$$
s=t_{o}-\theta_{\tau} \varrho^{p} .
$$

By definition of $\tau$ and (7.20), we find that

$$
\tau<s<t_{o} \quad \text { and } \quad u\left(x_{o}, s\right)<c_{1} \gamma^{c_{2}-1} u\left(x_{o}, t_{o}\right) .
$$

In the following we show by contradiction that

$$
\sup _{B_{\varrho}\left(x_{o}\right)} u(y, s)<c_{1} \gamma^{c_{2}-1} u\left(x_{o}, t_{o}\right) .
$$

Indeed, otherwise by the continuity of $u$ there existed $y \in B_{\varrho}\left(x_{o}\right)$ with $u(y, s)=$ $c_{1} \gamma^{c_{2}-1} u\left(x_{o}, t_{o}\right)$. For $\theta_{s}:=c_{o}^{d} u(y, s)^{-d}$ we have that $\left(s-2 \theta_{s} \varrho^{p}, s+2 \theta_{s} \varrho^{p}\right) \subset\left(t_{o}-\right.$ $\left.4 \theta \varrho^{p}, t_{o}+4 \theta \varrho^{p}\right)$. Thus, applying the forward Harnack inequality with $(y, s)$ instead of $\left(x_{o}, t_{o}\right)$ leads to

$$
\|F\|_{L^{\sigma}\left(\Omega_{T}\right)} \varrho^{p-1-\frac{n+p}{\sigma}} \geq \frac{1}{\gamma} u(y, s)^{d+1-\frac{d}{\sigma}}>\frac{1}{\gamma} u\left(x_{o}, t_{o}\right)^{d+1-\frac{d}{\sigma}},
$$

which contradicts (7.17), or

$$
u(y, s) \leq \gamma \inf _{B_{\varrho}(y)} u\left(\cdot, s+\theta_{s} \varrho^{p}\right) .
$$

Since $s+\theta_{s} \varrho^{p}=t_{o}$ and $y \in B_{\varrho}\left(x_{o}\right)$, we obtain the contradiction

$$
c_{1} \gamma^{c_{2}-1} u\left(x_{o}, t_{o}\right)=u(y, s) \leq \gamma u\left(x_{o}, t_{o}\right) .
$$

Therefore (7.21) is valid. Recalling the definition of $s$, we conclude that the desired backwards Harnack inequality is in force also in this case. This finishes the proof of inequality (7.16) and thus the proof of Theorem 1.2.

Acknowledgements V. Bögelein has been supported by the FWF-Project P31956-N32 "Doubly nonlinear evolution equations". A. Herán has been supported by the DFG-Project HA 7610/1-1 "Existenz- und Regularitätsaussagen für parabolische Quasiminimierer auf metrischen Maßräumen". L. Schätzler has been supported by Studienstiftung des deutschen Volkes.

Funding Open access funding provided by Austrian Science Fund (FWF). 
Open Access This article is licensed under a Creative Commons Attribution 4.0 International License, which permits use, sharing, adaptation, distribution and reproduction in any medium or format, as long as you give appropriate credit to the original author(s) and the source, provide a link to the Creative Commons licence, and indicate if changes were made. The images or other third party material in this article are included in the article's Creative Commons licence, unless indicated otherwise in a credit line to the material. If material is not included in the article's Creative Commons licence and your intended use is not permitted by statutory regulation or exceeds the permitted use, you will need to obtain permission directly from the copyright holder. To view a copy of this licence, visit http://creativecommons.org/licenses/by/4.0/.

\section{References}

1. Acerbi, E., Fusco, N.: Regularity for minimizers of nonquadratic functionals: the case $1<p<2$. J. Math. Anal. Appl. 140(1), 115-135 (1989)

2. Bögelein, V., Lukkari, T., Scheven, C.: Hölder regularity for degenerate parabolic obstacle problems. Ark. Mat. 55(1), 1-39 (2017)

3. Bögelein, V., Duzaar, F., Kinnunen, J., Scheven, C.: Higher integrability for doubly nonlinear parabolic systems. J. Math. Pures Appl. 143, 31-72 (2020)

4. Bögelein, V., Duzaar, F., Korte, R., Scheven, C.: The higher integrability of weak solutions of porous medium systems. Adv. Nonlinear Anal. 8(1), 1004-1034 (2019)

5. Bögelein, V., Duzaar, F., Marcellini, P.: Parabolic systems with p; q-growth: a variational approach. Arch. Ration. Mech. Anal. 210(1), 219-267 (2013)

6. Bögelein, V., Lehtelä, P., Sturm, S.: Regularity of weak solutions and supersolutions to the porous medium equation. Nonlinear Anal. 185, 49-67 (2019)

7. DiBenedetto, E.: Intrinsic Harnack type inequalities for solutions of certain degenerate parabolic equations. Arch. Ration. Mech. Anal. 100(2), 129-147 (1988)

8. DiBenedetto, E.: Degenerate parabolic equations, Springer Science \& Business Media (1993)

9. DiBenedetto, E., Gianazza, U., Vespri, V.: Harnack estimates for quasi-linear degenerate parabolic differential equations. Acta Math. 200(2), 181-209 (2008)

10. DiBenedetto, E., Gianazza, U., Vespri, V.: Harnack's Inequality for Degenerate and Singular Parabolic Equations. Springer Monographs in Mathematics. Springer, New York (2012)

11. Düzgün, F.G., Fornaro, S., Vespri, V.: Interior Harnack estimates: the state-of-the-art for quasilinear singular parabolic equations. Milan J. Math. 83(2), 371-395 (2015)

12. Fornario, S., Sosio, M.: Intrinsic Harnack estimates for some doubly nonlinear degenerate parabolic equations. Adv. Diff. Equ. 13(1-2), 139-168 (2008)

13. Fornario, S., Sosio, M., Vespri, V.: Harnack type inequalities for some doubly nonlinear singular parabolic equations. Discr. Cont. Dyn. Syst. A 35(12), 5909-5926 (2015)

14. Gianazza, U., Vespri, V.: A Harnack inequality for solutions of doubly nonlinear parabolic equations. J. Appl. Funct. Anal. 1(3), 271-284 (2006)

15. Giaquinta, M., Modica, G.: Remarks on the regularity of the minimizers of certain degenerate functionals. Manuscripta Math. 57(1), 55-99 (1986)

16. Hadamard, J.: Extension à l'èquation de la chaleur d'un théorème de A. Harnack. Rend. Circ. Mat. Palermo 2(3), 337-346 (1954)

17. Ivanov, A.V.: Hölder estimates for a natural class of equations of fast diffusion type, (Russian) Zap. Nauchn. Sem. S.-Peterburg. Otdel. Mat. Inst. Steklov. (POMI) 229, : Chisl. Metody i Voprosy Organ. Vychisl. 11(29-62), 322 (1995)

18. Ivanov, A.V.: Hölder estimates for a natural class of equations of fast diffusion type, translation in. J. Math. Sci. (New York) 89(6), 1607-1630 (1998)

19. Ivanov, A.V.: Hölder estimates for equations of slow and normal diffusion type, (Russian) Zap. Nauchn. Sem. S.-Peterburg. Otdel. Mat. Inst. Steklov. (POMI) 215, : Differentsial'naya Geom. Gruppy Li i Mekh. 14(130-136), 311 (1994)

20. Ivanov, A.V.: Hölder estimates for equations of slow and normal diffusion type, translation in. J. Math. Sci. (New York) 85(1), 1640-1644 (1997)

21. Kinnunen, J., Lindqvist, P.: Pointwise behaviour of semicontinuous supersolutions to a quasilinear parabolic equation. Ann. Mat. Pura Appl. 185(3), 411-435 (2006)

22. Kinnunen, J., Kuusi, T.: Local behaviour of solutions to doubly nonlinear parabolic equations Math. Annals 337(3), 705-728 (2007)

23. Kuusi, T.: Harnack estimates for weak supersolutions to nonlinear degenerate parabolic equations. Ann. Sc. Norm. Super. Pisa Cl. Sci. (5) (4), 673-716 (2008) 
24. Li, Q.: Weak Harnack estimates for supersolutions to doubly degenerate parabolic equations. Nonlinear Anal. 170, 88-122 (2018)

25. Moser, J.: On Harnack's theorem for elliptic differential equations. Comm. Pure Appl. Math. 14, 577-591 (1961)

26. Moser, J.: A Harnack inequality for parabolic differential equations. Comm. Pure Appl. Math. 17, 101-134 (1964)

27. Pini, B.: Sulla soluzione generalizzata di Wiener per il primo problema di valori al contorno nel caso parabolico. Rend. Sem. Mat. Univ. Padova 23, 422-434 (1954)

28. Porzio, M.M., Vespri, V.: Hölder estimates for local solutions of some doubly nonlinear degenerate parabolic equations. J. Diff. Equ. 103(1), 146-178 (1993)

29. Serrin, J.: Local behavior of solutions of quasi-linear equations. Acta Math. 111, 247-302 (1964)

30. Singer, T., Vestberg, M.: Local boundedness of weak solutions to the diffusive wave approximation of the shallow water equations. J. Diff. Equ. 266(6), 3014-3033 (2019)

31. Trudinger, N.S.: On Harnack type inequalities and their application to quasilinear elliptic equations. Comm. Pure Appl. Math. 20, 721-747 (1967)

32. Trudinger, N.S.: Pointwise estimates and quasilinear parabolic equations. Comm. Pure Appl. Math. 21(3), 205-226 (1968)

33. Vespri, V.: Harnack type inequalities for solutions of certain doubly nonlinear parabolic equations. J. Math. Anal. Appl. 181(1), 104-131 (1994)

34. Vespri, V., Vestberg, M.: An extensive study of the regularity of solutions to doubly singular equations. Adv. Calc. Var. (2020). https://doi.org/10.1515/acv-2019-0102

Publisher's Note Springer Nature remains neutral with regard to jurisdictional claims in published maps and institutional affiliations. 